\title{
Supersymmetry, Localization and Quantum Entropy Function
}

\author{
Nabamita Banerjee, Shamik Banerjee, Rajesh Kumar Gupta, \\ Ipsita Mandal and Ashoke Sen \\ Harish-Chandra Research Institute \\ Chhatnag Road, Jhusi, Allahabad 211019, INDIA \\ E-mail: nabamita, bshamik, rajesh, ipsita, sen at hri.res.in
}

\begin{abstract}
$A d S_{2} / C F T_{1}$ correspondence leads to a prescription for computing the degeneracy of black hole states in terms of path integral over string fields living on the near horizon geometry of the black hole. In this paper we make use of the enhanced supersymmetries of the near horizon geometry and localization techniques to argue that the path integral receives contribution only from a special class of string field configurations which are invariant under a subgroup of the supersymmetry transformations. We identify saddle points which are invariant under this subgroup. We also use our analysis to show that the integration over infinite number of zero modes generated by the asymptotic symmetries of $A d S_{2}$ generate a finite contribution to the path integral.
\end{abstract}




\section{Contents}

1 Introduction

2

2 Symmetries of Euclidean $A d S_{2} \times S^{2} \quad 4$

3 Localization $\quad 8$

4 Integrating Over the Orbit of the Superconformal Current Algebra $\quad 14$

5 Examples of $H_{1}$-invariant Saddle Points $\quad 15$

6 Comments $\quad 19$

A Killing Spinors in Six Dimensional Supergravity on $S^{1} \times \widetilde{S}^{1} \times A d S_{2} \times S^{2} \quad 20$

\section{Introduction}

Supersymmetric extremal black holes typically have an $A d S_{2}$ factor in their near horizon geometry. Based on $A d S_{2} / C F T_{1}$ correspondence refs. [1,2,3] proposed a definite relation between the degeneracy $d_{\text {hor }}$ associated with the black hole horizon and the partition function of string theory on the near horizon geometry. This relation takes the form:

$$
d_{\text {hor }}=\left\langle\exp \left[-i q_{i} \oint d \theta A_{\theta}^{(i)}\right]\right\rangle_{A d S_{2}}^{\text {finite }}
$$

where \langle\rangle$_{A d S_{2}}$ denotes the unnormalized path integral over all the fields in string theory, weighted by $e^{-A}$ where $A$ is the Euclidean action, with the boundary condition that asymptotically the field configuration approaches the near horizon geometry of the black hole containing an $A d S_{2}$ factor. $\left\{A^{(i)}\right\}$ denote the set of all $U(1)$ gauge fields of string theory living on the $A d S_{2}$ component of the near horizon geometry, $q_{i}$ is the $i$-th electric charge carried by the black hole and $\oint d \theta A_{\theta}^{(i)}$ denotes the integral of the $i$-th gauge field along the boundary of $A d S_{2} . q_{i}$ in particular also includes the angular momentum of the black hole [4]. The superscript ' $f$ inite' refers to the finite part of the amplitude defined as follows. If we represent $A d S_{2}$ as the Poincare disk, regularize the infinite volume of $A d S_{2}$ by putting an infrared cut-off and denote by $L$ the length of the boundary of this regulated $A d S_{2}$, then for large cutoff, 1.e. large $L$, the amplitude has the form $e^{C L+\mathcal{O}\left(L^{-1}\right)} \times \Delta$ where $C$ and $\Delta$ are $L$-independent constants. 
The finite part of the amplitude is defined to be the constant $\Delta$, and has been named the quantum entropy function.

In computing the path integral we need to keep the asymptotic values of electric fields fixed 1 and integrate over the constant modes of the gauge fields. As a result the path integral directly computes the degeneracy in a fixed charge sector, 1.e. entropy in the microcanonical ensemble, as opposed to a partition function. Due to the same reason it also computes the degeneracy in a fixed angular momentum sector. This allows us to compute the index in terms of the degeneracy $d_{h o r}$ which can then be compared with the result for the microscopic degeneracy [3]. It was found in [1,5,6] that in the semiclassical limit the result of this path integral reproduces correctly the exponential of the Wald entropy [7, 8, 9, 10] via the entropy function formalism [11,12] even after taking into account higher derivative corrections to the classical action.

In four space-time dimensions supersymmetry requires the black holes to be spherically symmetric, and as a consequence the near horizon geometry has an $A d S_{2} \times S^{2}$ factor. For $1 / 8$ BPS black holes in $\mathcal{N}=8$ supersymmetric theories, $1 / 4$ BPS black holes in $\mathcal{N}=4$ supersymmetric theories and $1 / 2$ BPS black holes in $\mathcal{N}=2$ supersymmetric theories, the $S L(2, \mathbb{R}) \times S O(3)$ isometry of the near horizon geometry gets enhanced to the $S U(1,1 \mid 2)$ supergroup. The goal of this paper will be to simplify the path integral over string fields appearing in the definition of (1.1) by making use of these isometries. In particular we shall use localization techniques $[13,14,15,16,17,18,19,20,21,22,23$, to show that the path integral receives contribution only from a special class of field configurations which preserve a particular subgroup of $S U(1,1 \mid 2)$.

The effect of localization in these theories have been studied earlier in 24] for analyzing the world-sheet instanton contributions. Our goal is quite different as we use it to classify string field configurations which could contribute to the path integral. Thus for example our analysis can be used to restrict the saddle points, 1.e. classical string field configurations, which contribute to the path integral over string fields. These are relevant for computing nonperturbative corrections to the quantum entropy function whereas the world-sheet instanton corrections are relevant only for string loop computation.

The rest of the paper is organised as follows. In $₫ 2$ we describe the algebra underlying the $S U(1,1 \mid 2)$ group and also the reality condition on the various generators required to represent

\footnotetext{
${ }^{1}$ These fixed values are determined in terms of $q_{i}$ by requiring that the boundary terms in the variation of the action cancel.
} 
the symmetries of the Euclidean near horizon geometry. In $\$ 3$ we use localization techniques developed in [18,19] to argue that the path integral receives contribution only from a special class of string field configurations invariant under a special subgroup $H_{1}$ of the $S U(1,1 \mid 2)$ group. In $\$ 4$ we use the results of $\$ 3$ to show that integration over the bosonic and fermion zero modes, generated by an infinte dimensional group of asymptotic symmetries, actually gives a finite result to the path integral. In $\$ 5$ we give some examples of $H_{1}$-invariant saddle points which contribute to the path integral. In $\$ 6$ we discuss possible application of our result to further simplify the analysis of quantum entropy function and also a possible application to computing the expectation values of circular 't Hooft - Wilson loop operators in superconformal gauge theories following [25]. In appendix A] we analyze the killing spinors in the near horizon geometry of a specific class of quarter BPS black holes in type IIB string theory compactified on $K 3 \times T^{2}$ and show that they indeed generate the $s u(1,1 \mid 2)$ algebra described in $\$ 2$,

\section{Symmetries of Euclidean $A d S_{2} \times S^{2}$}

We begin by writing down the global part of the $\mathcal{N}=4$ superconformal algebra in $1+1$ dimensions. Its non-vanishing commutators are

$$
\begin{aligned}
& {\left[L_{m}, L_{n}\right]=(m-n) L_{m+n},} \\
& {\left[J^{3}, J^{ \pm}\right]= \pm J^{ \pm}, \quad\left[J^{+}, J^{-}\right]=2 J^{3},} \\
& {\left[L_{n}, G_{r}^{\alpha \pm}\right]=\left(\frac{n}{2}-r\right) G_{r+n}^{\alpha \pm},} \\
& {\left[J^{3}, G_{r}^{\alpha \pm}\right]= \pm \frac{1}{2} G_{r}^{\alpha \pm}, \quad\left[J^{ \pm}, G_{r}^{\alpha \mp}\right]=G_{r}^{\alpha \pm},} \\
& \left\{G_{r}^{+\alpha}, G_{s}^{-\beta}\right\}=2 \epsilon^{\alpha \beta} L_{r+s}-2(r-s)\left(\epsilon \sigma^{i}\right)_{\beta \alpha} J^{i} \\
& \epsilon^{+-}=-\epsilon^{-+}=1, \quad \epsilon^{++}=\epsilon^{--}=0, \quad m, n=0, \pm 1, \quad r, s= \pm \frac{1}{2}, \quad \alpha, \beta= \pm .
\end{aligned}
$$

The right superscript of $G_{r}$ denotes the transformation properties under the $\mathrm{SU}(2)$ current algebra whose zero modes are denoted by $\left(J^{3}, J^{ \pm}=J^{1} \pm i J^{2}\right)$. There is also an $S U(2)$ group acting on the left superscript. This describes an outer automorphism of the supersymmetry algebra but is not in general a symmetry of the theory. In eq.(2.1) and in the rest of this paper we shall use the convention that when an equation involves \pm or $\mp$, it represents only two equations - first one being obtained by choosing the upper sign in all the terms and the 
second one obtained by choosing the lower sign in all the terms. The supergroup generated by the algebra (2.1) is known as $S U(1,1 \mid 2)$.

In the above, the action of the Virasoro generators on the coordinate $u$ labelling the upper half plane (UHP) is of the form

$$
L_{n}=-u^{n+1} \partial_{u}-\bar{u}^{n+1} \partial_{\bar{u}} .
$$

However while describing symmetries of the Euclidean $A d S_{2} \times S^{2}$, which is isomorphic to $U H P \times S^{2}$ it is more natural to use the Virasoro generators

$$
L_{n}=-\left(i u^{n+1} \partial_{u}+i \bar{u}^{n+1} \partial_{\bar{u}}\right),
$$

so that the elements of $\mathrm{SL}(2, \mathbb{R})$ can be labelled as $\exp \left(i s_{n} L_{n}\right)$ with real parameters $s_{n}$, just as $\exp \left(i t_{i} J^{i}\right)$ labels an element of the $S U(2)$ group for real $t_{i}$. The corresponding algebra is obtained from (2.1) by scaling the Virasoro generators by $i$. For later convenience we shall also multiply $G_{r}^{+\alpha}$ by $e^{i s_{0}}$ and $G_{r}^{-\alpha}$ by $i e^{-i s_{0}}$ for some arbitrary fixed phase $e^{i s_{0}}$. This gives 2

$$
\begin{aligned}
& {\left[L_{m}, L_{n}\right]=i(m-n) L_{m+n},} \\
& {\left[J^{3}, J^{ \pm}\right]= \pm J^{ \pm}, \quad\left[J^{+}, J^{-}\right]=2 J^{3},} \\
& {\left[L_{n}, G_{r}^{\alpha \pm}\right]=i\left(\frac{n}{2}-r\right) G_{r+n}^{\alpha \pm},} \\
& {\left[J^{3}, G_{r}^{\alpha \pm}\right]= \pm \frac{1}{2} G_{r}^{\alpha \pm}, \quad\left[J^{ \pm}, G_{r}^{\alpha \mp}\right]=G_{r}^{\alpha \pm},} \\
& \left\{G_{r}^{+\alpha}, G_{s}^{-\beta}\right\}=2 \epsilon^{\alpha \beta} L_{r+s}-2 i(r-s)\left(\epsilon \sigma^{i}\right)_{\beta \alpha} J^{i} \\
& \epsilon^{+-}=-\epsilon^{-+}=1, \quad \epsilon^{++}=\epsilon^{--}=0, \quad m, n=0, \pm 1, \quad r, s= \pm \frac{1}{2}, \quad \alpha, \beta= \pm .
\end{aligned}
$$

Often it is convenient to represent $A d S_{2}$ as a unit disk labelled by a coordinate $w$ related to $u$ via:

$$
w=\frac{1+i u}{1-i u} .
$$

In the $w$ coordinate system

$$
L_{n}=\frac{i}{2}\left[i^{n}(1+w)^{1-n}(1-w)^{1+n} \partial_{w}+\text { c.c. }\right] .
$$

On the other hand the action of the $J^{i}$ s on the stereographic coordinate $z$ of the sphere $S^{2}$ takes the form

$$
J^{3}=\left(z \partial_{z}-\bar{z} \partial_{\bar{z}}\right), \quad J^{+}=z^{2} \partial_{z}+\partial_{\bar{z}}, \quad J^{-}=-\bar{z}^{2} \partial_{\bar{z}}-\partial_{z} .
$$

\footnotetext{
${ }^{2}$ Note that while computing the commutators we regard the action of the generators as active transformation.
} 
It is easy to see that the $A d S_{2} \times S^{2}$ metric

$$
d s^{2}=4 v \frac{d w d \bar{w}}{(1-\bar{w} w)^{2}}+4 u \frac{d z d \bar{z}}{(1+\bar{z} z)^{2}}
$$

where $u$ and $v$ are constants, is invariant under these transformations. Making the coordinate transformations

$$
w=\tanh \frac{\eta}{2} e^{i \theta}, \quad z=\tan \frac{\psi}{2} e^{i \phi},
$$

we can express the metric (2.8) as

$$
d s^{2}=v\left(d \eta^{2}+\sinh ^{2} \eta d \theta^{2}\right)+u\left(d \psi^{2}+\sin ^{2} \psi d \phi^{2}\right) .
$$

This form of the $A d S_{2} \times S^{2}$ metric coincides with the one given in (A.26). In appendix $\mathrm{A}$ we have shown that the full $S U(1,1 \mid 2)$ group generated by $\widehat{L}_{0}, \widehat{L}_{ \pm}, J^{3}, J^{ \pm}$and $\widehat{G}_{\gamma}^{\alpha \beta}$ describes a symmetry of the near horizon $A d S_{2} \times S^{2}$ geometry of Euclidean BPS black holes.

We now define

$$
\widehat{L}_{0}=\frac{1}{2}\left(L_{1}+L_{-1}\right), \quad \widehat{L}_{ \pm}=L_{0} \pm \frac{i}{2}\left(L_{1}-L_{-1}\right), \quad \widehat{G}_{ \pm}^{\alpha \beta}=G_{1 / 2}^{\alpha \beta} \mp i G_{-1 / 2}^{\alpha \beta} .
$$

From eqs.(2.6), (2.11) we see that the action of $\widehat{L}_{0}, \widehat{L}_{ \pm}$on the $w$-plane is given by

$$
\widehat{L}_{0}=\left(w \partial_{w}-\bar{w} \partial_{\bar{w}}\right), \quad \widehat{L}_{+}=-i\left(w^{2} \partial_{w}-\partial_{\bar{w}}\right), \quad \widehat{L}_{-}=i\left(\partial_{w}-\bar{w}^{2} \partial_{\bar{w}}\right) .
$$

This shows that $\widehat{L}_{0}$ has the interpretation of the generator of rotation about the origin in the $w$-plane. In terms of these new generators the non-vanishing (anti-)commutators of the $s u(1,1 \mid 2)$ algebra take the form

$$
\begin{aligned}
& {\left[\widehat{L}_{0}, \widehat{L}_{ \pm}\right]= \pm \widehat{L}_{ \pm}, \quad\left[\widehat{L}_{+}, \widehat{L}_{-}\right]=-2 \widehat{L}_{0}} \\
& {\left[J^{3}, J^{ \pm}\right]= \pm J^{ \pm}, \quad\left[J^{+}, J^{-}\right]=2 J^{3},} \\
& {\left[\widehat{L}_{0}, \widehat{G}_{ \pm}^{\alpha \beta}\right]= \pm \frac{1}{2} \widehat{G}_{ \pm}^{\alpha \beta}, \quad\left[\widehat{L}_{ \pm}, \widehat{G}_{\mp}^{\alpha \beta}\right]=-i \widehat{G}_{ \pm}^{\alpha \beta},} \\
& {\left[J^{3}, \widehat{G}_{\beta}^{\alpha \pm}\right]= \pm \frac{1}{2} \widehat{G}_{\beta}^{\alpha \pm}, \quad\left[J^{ \pm}, \widehat{G}_{\beta}^{\alpha \mp}\right]=\widehat{G}_{\beta}^{\alpha \pm},} \\
& \left\{\widehat{G}_{ \pm}^{+\alpha}, \widehat{G}_{\mp}^{-\beta}\right\}=4 \epsilon^{\alpha \beta} \widehat{L}_{0} \pm 4\left(\epsilon \sigma^{i}\right)_{\beta \alpha} J^{i}, \quad\left\{\widehat{G}_{ \pm}^{+\alpha}, \widehat{G}_{ \pm}^{-\beta}\right\}=\mp 4 i \epsilon^{\alpha \beta} \widehat{L}_{ \pm} .
\end{aligned}
$$

Note that an element of the form $\exp \left[i\left(\xi^{0} \widehat{L}_{0}+\xi^{+} \widehat{L}_{+}+\xi^{-} \widehat{L}_{-}+\eta_{3} J^{3}+\eta_{+} J^{+}+\eta_{-} J^{-}\right)\right]$will be an element of the $S L(2, \mathbb{R}) \times S U(2)$ group if we have

$$
\left(\xi^{0}\right)^{*}=\xi^{0}, \quad\left(\xi^{ \pm}\right)^{*}=\xi^{\mp}, \quad\left(\eta_{3}\right)^{*}=\eta_{3}, \quad\left(\eta_{ \pm}\right)^{*}=\eta_{\mp} .
$$


We shall call these the reality conditions on the bosonic generators. We shall now impose a similar reality condition on the fermionic generators, 1.e. specify the condition on the complex grassman parameters $\theta_{\alpha \beta}^{\gamma}$ under which $\exp \left[i \theta_{\alpha \beta}^{\gamma} G_{\gamma}^{\alpha \beta}\right]$ describes an element of the $S U(1,1 \mid 2)$ group. Any such rule must be compatible with the requirement that if $\exp \left(i T_{1}\right)$ and $\exp \left(i T_{2}\right)$ are two elements of the $S U(1,1 \mid 2)$ group, then $\exp \left(\left[T_{1}, T_{2}\right]\right)$ must also be an element of this group. The following constraint on $\theta_{\alpha \beta}^{\gamma}$ is compatible with this rule 3

$$
\left(\theta_{\alpha \beta}^{\gamma}\right)^{*}=\epsilon^{\alpha \alpha^{\prime}} \epsilon^{\beta \beta^{\prime}} \theta_{\alpha^{\prime} \beta^{\prime}}^{-\gamma}
$$

Equivalently we can say that

$$
\exp \left[i \theta\left(\widehat{G}_{\gamma}^{\alpha \beta}+\epsilon^{\alpha \alpha^{\prime}} \epsilon^{\beta \beta^{\prime}} \widehat{G}_{-\gamma}^{\alpha^{\prime} \beta^{\prime}}\right)\right] \text { and } \exp \left[\theta\left(\widehat{G}_{\gamma}^{\alpha \beta}-\epsilon^{\alpha \alpha^{\prime}} \epsilon^{\beta \beta^{\prime}} \widehat{G}_{-\gamma}^{\alpha^{\prime} \beta^{\prime}}\right)\right] \text {, }
$$

are elements of $S U(1,1 \mid 2)$ for real $\theta$. We shall proceed with this choice. If we now define

$$
\begin{aligned}
& Q_{1}=\widehat{G}_{+}^{++}+\widehat{G}_{-}^{--}, \quad Q_{2}=-i\left(\widehat{G}_{+}^{++}-\widehat{G}_{-}^{--}\right), \\
& Q_{3}=-i\left(\widehat{G}_{+}^{-+}+\widehat{G}_{-}^{+-}\right), \quad Q_{4}=\widehat{G}_{+}^{-+}-\widehat{G}_{-}^{+-}, \\
& \widetilde{Q}_{1}=\widehat{G}_{-}^{++}+\widehat{G}_{+}^{--}, \quad \widetilde{Q}_{2}=-i\left(\widehat{G}_{-}^{++}-\widehat{G}_{+}^{--}\right), \\
& \widetilde{Q}_{3}=-i\left(\widehat{G}_{-}^{-+}+\widehat{G}_{+}^{+-}\right), \quad \widetilde{Q}_{4}=\widehat{G}_{-}^{-+}-\widehat{G}_{+}^{+-},
\end{aligned}
$$

then $\exp \left(i \theta Q_{i}\right)$ and $\exp \left(i \theta \widetilde{Q}_{i}\right)$ are elements of $S U(1,1 \mid 2)$ for real $\theta$. In that case we have $\left\{Q_{i}, Q_{j}\right\}=8 \delta_{i j}\left(\widehat{L}_{0}-J^{3}\right), \quad\left\{\widetilde{Q}_{i}, \widetilde{Q}_{j}\right\}=8 \delta_{i j}\left(\widehat{L}_{0}+J^{3}\right), \quad\left[\widehat{L}_{0}-J^{3}, Q_{i}\right]=0, \quad\left[\widehat{L}_{0}+J^{3}, \widetilde{Q}_{i}\right]=0$.

Besides this, $\left\{Q_{i}, \widetilde{Q}_{j}\right\}$ are given by linear combinations of $J^{ \pm}$and $\widehat{L}_{ \pm},\left[\widehat{L}_{0}-J^{3}, \widetilde{Q}_{i}\right]$ are given by linear combinations of $\widetilde{Q}_{i},\left[\widehat{L}_{0}+J^{3}, Q_{i}\right]$ are given by linear combinations of $Q_{i},\left[J^{ \pm}, Q_{i}\right]$ and $\left[\widehat{L}_{ \pm}, Q_{i}\right]$ are given by linear combinations of $\widetilde{Q}_{i}$ and $\left[J^{ \pm}, \widetilde{Q}_{i}\right],\left[\widehat{L}_{ \pm}, \widetilde{Q}_{i}\right]$ are given by linear combinations of $Q_{i}$. Precise form of these relations can be determined from (2.13) and (2.17), but we shall not write them down explicitly.

Given the reality condition on the various generators, we can label an element of $S U(1,1 \mid 2)$ as

$$
\begin{aligned}
g\left(\xi, \bar{\xi}, \eta, \bar{\eta}, \sigma, \widetilde{\sigma},\left\{\theta_{\alpha \beta}\right\},\left\{\chi_{i}\right\}\right)= & \exp \left[i\left\{\bar{\xi} \widehat{L}_{+}+\xi \widehat{L}_{-}+\bar{\eta} J^{+}+\eta J^{-}+\theta_{\alpha+} \widehat{G}_{-}^{\alpha+}+\theta_{\alpha-} \widehat{G}_{+}^{\alpha-}\right\}\right] \\
& \times \exp \left[i \sigma\left(\widehat{L}_{0}+J^{3}\right)\right] \times \exp \left[i\left\{\sum_{k=1}^{4} \chi_{k} Q_{k}+\widetilde{\sigma}\left(\widehat{L}_{0}-J^{3}\right)\right\}\right],
\end{aligned}
$$

${ }^{3}$ We need to remember that if $\theta_{1}$ and $\theta_{2}$ are real grassman parameters then $\left(\theta_{1} \theta_{2}\right)^{*}=\theta_{2} \theta_{1}=-\theta_{1} \theta_{2}$. 
where $\xi, \eta$ are complex bosonic parameters, $\sigma, \widetilde{\sigma}$ are real bosonic parameters, $\chi_{i}$ are real grassman parameters and $\theta_{\alpha \beta}$ are complex grassman parameters satisfying the reality condition

$$
\left(\theta_{\alpha \beta}\right)^{*}=\epsilon^{\alpha \alpha^{\prime}} \epsilon^{\beta \beta^{\prime}} \theta_{\alpha^{\prime} \beta^{\prime}} .
$$

Let us also denote by $H_{0}$ the subgroup of $S U(1,1 \mid 2)$ generated by

$$
\widehat{L}_{0}-J^{3}, \quad Q_{1}, \quad Q_{2}, \quad Q_{3}, \quad Q_{4} .
$$

The non-vanishing (anti-)commutators of $H_{0}$ are

$$
\left[\widehat{L}_{0}-J^{3}, Q_{i}\right]=0, \quad\left\{Q_{i}, Q_{j}\right\}=8 \delta_{i j}\left(\widehat{L}_{0}-J^{3}\right) .
$$

Then in (2.19) the parameters $\widetilde{\sigma}$ and $\left\{\chi_{i}\right\}$ parametrize an element of $H_{0}$ and the parameters $\sigma, \xi, \eta$ and $\theta_{\alpha \beta}$ parametrize the coset $G / H_{0}$.

Finally another subgroup of $S U(1,1 \mid 2)$ (and $H_{0}$ ) that will play an important role in our analysis is the subgroup $H_{1}$ generated by $Q_{1}$ and $\left(\widehat{L}_{0}-J^{3}\right)$.

\section{Localization}

In computing the quantum entropy function, - the partition function of string theory on the near horizon geometry of the black hole - we need to integrate over all string field configurations. In order to carry out the path integral, which involves integration over infinite number of modes, it will be useful to fix the order in which we carry out the integration. We shall adopt the following definition of the path integral: first we shall integrate over the orbits of the subgroup $H_{1}$ generated by $Q_{1}$ and $\left(\widehat{L}_{0}-J^{3}\right)$, then over the orbits generated by the others $Q_{i}$ 's belonging to the subgroup $H_{0}$ and then carry out the integration over the remaining variables in some order. As we shall see this definition will allow us to arrive at simple results on which configurations could contribute to the path integral. Our approach follows closely that of [18]. Throughout this analysis we shall implicitly assume that the theory admits a formalism in which at least the $H_{1}$ subalgebra of the $s u(1,1 \mid 2)$ algebra, generated by $Q_{1}$ and $\left(\widehat{L}_{0}-J^{3}\right)$, is realized off-shell. It may be possible to achieve this by generalizing the trick used in [21] for $\mathcal{N}=4$ supersymmetric gauge theories. Finally we shall ignore the various issues related to gauge fixing. For supersymmetric gauge theories in four dimensions gauge fixing introduces various subtleties in the proof of localization [21. However eventually these can be overcome, and we shall assume that similar results will hold for supergravity as well. 
Formally the division of the path integral into orbits of $H_{1}$ and directions transverse to these orbits can be done by manipulating the integral using Fadeev-Popov method. 4 By expressing an element of $H_{1}$ as

$$
h=\exp \left(i \alpha Q_{1}+i \beta\left(\widehat{L}_{0}-J^{3}\right)\right)
$$

we can express the path integral as

$$
\left[\int d h\right]\left[\left.\int e^{-A}\left(\prod_{a} \delta\left(F^{a}\right)\right) \operatorname{sdet} \frac{\delta F_{\vec{\tau}}^{a}}{\partial \tau^{b}}\right|_{\vec{\tau}=0}\right]
$$

where $\int d h$ denotes integration over the group $H_{1}$ with Haar measure, $A$ is the Euclidean action $\sqrt[5]{5} F^{a}$ are a pair of 'gauge fixing functionals' of the field configuration, $\vec{\tau}$ denote collectively the parameters $(\alpha, \beta)$ labelling the elements of the group $H_{1}$ and $F_{\vec{\tau}}^{a}$ is the transform of $F^{a}$ by the group element corresponding to the parameters $\vec{\tau}$. We now note that the integration over $H_{1}$ has a bosonic direction $\beta$ which parametrizes a compact $U(1)$ group and hence gives a finite result, and a fermionic direction $\alpha$. By the standard rules of integration over grassman parameters the fermionic integral gives a zero, making the whole integral vanish.

This argument breaks down around a configuration $\Phi$ which is invariant under a subgroup of $H_{1}$, since the matrix $\left(\delta F_{\vec{\tau}}^{a} / \delta \tau^{b}\right)$ in $(\underline{3.2})$ becomes degenerate at this point. In this case we proceed as follows. First of all note that a subgroup of $H_{1}$ can either be the whole of $H_{1}$ or the $U(1)$ group generated by $\left(\widehat{L}_{0}-J^{3}\right)$. However if $\Phi$ is invariant only under $\left(\widehat{L}_{0}-J^{3}\right)$, then the zero eigenvector of the matrix $\delta F_{\vec{\tau}}^{a} /\left.\partial \tau^{b}\right|_{\vec{\tau}=0}$ is along the bosonic direction corresponding to the $U(1)$ transformation. This makes the sdet factor in (3.2) vanish on the configuration $\Phi$ but does not generate any divergence in the integrand. Hence our earlier argument can still be applied to show that the $\int d h$ factor makes the integral vanish. Thus the configuration $\Phi$ must be invariant under both $Q_{1}$ and $\left(\widehat{L}_{0}-J^{3}\right)$. This allows us to choose the coordinates of the configuration space, measuring fluctuations around the configuration $\Phi$, as follows. First by Fourier decomposing these fluctuations in the $(\theta-\phi)$ coordinates we can choose them to be eigenvectors of $\left(\widehat{L}_{0}-J^{3}\right)$ with definite eigenvalues $m \in \mathbb{Z}$. For example for a scalar field a deformation of the form $e^{i m(\theta-\phi) / 2} f(\theta+\phi, r, \psi)$ for any arbitrary function $f$ will have this property. Let us parametrize the set of all such bosonic fluctuations by coordinates $z_{(m)}^{s}$. The

\footnotetext{
${ }^{4}$ Unlike in the case of a gauge symmetry here we do not divide the path integral by the volume of the 'gauge group' $H_{1}$. The rest of the manipulation proceeds exactly as in the case of a gauge theory.

${ }^{5}$ We are including in $A$ the bulk and the boundary contributions to the action including the $i \oint \vec{q} \cdot \vec{A}$ term that is necessary to make the path integral well defined [1,3]. We shall also be implicitly assuming that the boundary terms have been chosen so that all the supersymmetries of the bulk theory are preserved.
} 
complex conjugate deformation, labelled by $z_{(m)}^{s *}$ will have $\left(\widehat{L}_{0}-J^{3}\right)$ eigenvalue $-m$. To avoid double counting we shall denote the fluctuations with positive $m$ by $z_{(m)}^{s}$ and fluctuations with negative $m$ by $z_{(m)}^{s *}$. As $s$ runs over different values, the parameters $z_{(m)}^{s}$ produce the complete set of bosonic deformations with $\left(\widehat{L}_{0}-J^{3}\right)$ eigenvalue $m$. Now for $m \neq 0$, the action of the generator $Q_{1}$ on such a bosonic deformation cannot vanish since $Q_{1}^{2}=4\left(\widehat{L}_{0}-J^{3}\right)$ acting on the fluctuation does not vanish. Instead this will generate a particular fermionic deformation with $\left(\widehat{L}_{0}-J^{3}\right)$ eigenvalue $m$. Let us denote the parameter associated with the fermionic deformation by $\zeta_{(m)}^{s}$. Finally we shall call the $m=0$ bosonic and fermionic modes collectively as $\vec{y}$. Since the original configuration $\Phi$ is the origin of the coordinate system, all the coordinates vanish at $\Phi$. We can now write 6

$$
Q_{1} z_{(m)}^{s}=\zeta_{(m)}^{s}, \quad Q_{1} \zeta_{(m)}^{s}=4 m z_{(m)}^{s}
$$

where the second equation follows from the fact that $\left(Q_{1}\right)^{2} z_{(m)}^{s}=4 m z_{(m)}^{s}$. Using the reality of the operator $\left(i \epsilon Q_{1}\right)$ and the rules for complex conjugation of grassman variables described in footnote 3, the complex conjugate relations of (3.3) can be expressed in the form 7

$$
Q_{1} z_{(m)}^{s *}=\zeta_{(m)}^{s *}, \quad Q_{1} \zeta_{(m)}^{s *}=-4 m z_{(m)}^{s *} .
$$

$\zeta_{(m)}^{s}$ for different values of $s$ give the complete set of fermionic deformations with $\left(\widehat{L}_{0}-J^{3}\right)$ eigenvalue $m$ and $\zeta_{(m)}^{s *}$ for different values of $s$ give the complete set of fermionic deformations with $\left(\widehat{L}_{0}-J^{3}\right)$ eigenvalue $-m$. To see this let us assume the contrary, 1.e. that there is a fermionic coordinate $\chi_{(m)}$ carrying $\widehat{L}_{0}-J^{3}$ eigenvalue $m$ that is linearly independent of the $\zeta_{(m)}^{s}$ 's (up to quadratic and higher powers of the other coordinates). Since the origin is $Q_{1}$ invariant, $Q_{1} \chi_{(m)}$ must vanish at the origin. On the other hand if $Q_{1} \chi_{(m)}$ is bilinear in the coordinates $\left(\left\{z_{(m)}^{s}\right\},\left\{z_{(m)}^{s *}\right\},\left\{\zeta_{(m)}^{s}\right\},\left\{\zeta_{(m)}^{s *}\right\}, \chi_{(m)}, \vec{y}\right)$ then it will be impossible to satisfy the $Q_{1}^{2} \chi_{(m)}=4 m \chi_{(m)}$ condition since the action of $Q_{1}$ on each of the coordinates produces a term linear and higher order in these coordinates. Thus $Q_{1} \chi_{(m)}$ must be a linear combinations of the complete set of bosonic coordinates $\left\{z_{(m)}^{s}\right\}$ carrying $\widehat{L}_{0}-J^{3}$ eigenvalue $m$ up to additional

\footnotetext{
${ }^{6}$ Our convention for defining the action of $Q_{1}$ on the parameters will be as follows. Take a general field configuration labelled by $\left(\left\{z_{(m)}^{s}\right\},\left\{\zeta_{(m)}^{s}\right\}, \vec{y}\right)$ and act on it by the transformation $\left(1+i \epsilon Q_{1}\right)$. The new configuration can be associated with a new set of values of the various parameters. We call the parameters associated with the new configuration as $\left(\left\{z_{(m)}^{s}+i \epsilon Q_{1} z_{(m)}^{s}\right\},\left\{\zeta_{(m)}^{s}+i \epsilon Q_{1} \zeta_{(m)}^{s}\right\}, \vec{y}+i \epsilon Q_{1} \vec{y}\right)$.

${ }^{7}$ To see this we can write $z_{(m)}^{s}=z_{(m) R}^{s}+i z_{(m) I}^{s}, \zeta_{(m)}^{s}=\zeta_{(m) R}^{s}+i \zeta_{(m) I}^{s}$ with real $z_{(m) R}^{s}, z_{(m) I}^{s}, \zeta_{(m) R}^{s}$ and $\zeta_{(m) I}^{s}$, and then compare the real and imaginary parts of (3.3) after multiplying both sides by $i \theta$, keeping in mind that the operator $i \theta Q$ for real grassman parameter $\theta$ takes a real variable to a real variable, and also that given two real grassman variables $\theta_{1}, \theta_{2}, \theta_{1} \theta_{2}$ is imaginary. Eq.(3.4) follows from this immediately.
} 
higher order terms in the coordinates. Applying $Q_{1}$ on either side we see that $\chi_{(m)}$ must be a linear combination of the coordinates $\zeta_{(m)}^{s}$ up to additional higher order terms, in contrary to our original assumption that $\chi_{(m)}$ is linearly independent of the other $\zeta_{(m)}^{s}$ 's.

The coordinates $\left(\left\{z_{(m)}^{s}\right\},\left\{z_{(m)}^{s *}\right\},\left\{\zeta_{(m)}^{s}\right\},\left\{\zeta_{(m)}^{s *}\right\}\right)$ will in particular include the deformations generated by the elements of $S U(1,1 \mid 2)$ outside the subgroup generated by the $Q_{i}$ 's and $\left(\widehat{L}_{0} \pm\right.$ $\left.J^{3}\right)$, since such deformations will carry non-zero $\widehat{L}_{0}-J^{3}$ charge. If for example we use the parametrization given in (2.19) for an element of $S U(1,1 \mid 2)$, then the parameters $\xi, \bar{\eta}$ and $\theta_{\alpha+}$ will carry $\left(\widehat{L}_{0}-J^{3}\right)$ eigenvalue +1 , and their complex conjugate parameters will carry $\left(\widehat{L}_{0}-J^{3}\right)$ eigenvalue -1 .

Now the path integral over the various fields can be regarded as integral over the parameters $z_{(m)}^{s}, z_{(m)}^{s *}, \zeta_{(m)}^{s}$ and $\zeta_{(m)}^{s *}$ for different values of $s$ and $m \neq 0$ together with integration over the variables $\vec{y}$. Thus we have an integral

$$
I=\int d \vec{y} \prod_{m>0, s} d z_{(m)}^{s} d z_{(m)}^{s *} d \zeta_{(m)}^{s} d \zeta_{(m)}^{s *} \mathcal{J} e^{-A}
$$

where $\mathcal{J}$ represents any measure factor which might arise from changing the integration variables to $\left(\vec{y}, \vec{z}, \vec{z}^{*}, \vec{\zeta}, \vec{\zeta}^{*}\right)$. We now deform this to another integral

$$
I(t)=\int d \vec{y} \prod_{m>0} d z_{(m)}^{s} d z_{(m)}^{s *} d \zeta_{(m)}^{s} d \zeta_{(m)}^{s *} \mathcal{J} e^{-A-t Q_{1} F},
$$

where $t$ is a positive real parameter and

$$
F=\sum_{m>0} \sum_{s} z_{(m)}^{s *} \zeta_{(m)}^{s}
$$

This gives

$$
Q_{1} F=\sum_{m>0} \sum_{s}\left[4 m z_{(m)}^{s *} z_{(m)}^{s}+\zeta_{(m)}^{s *} \zeta_{(m)}^{s}\right] .
$$

Furthermore, since by construction $F$ is invariant under $\left(\widehat{L}_{0}-J^{3}\right)$, we have

$$
Q_{1}^{2} F=0 .
$$

This equation, together with the supersymmetry invariance of the action $\left(Q_{1} A=0\right)$ can be used to get

$$
\begin{aligned}
\partial_{t} I(t) & =\int d \vec{y} \prod_{m>0} d z_{(m)}^{s} d z_{(m)}^{s *} d \zeta_{(m)}^{s} d \zeta_{(m)}^{s *} \mathcal{J}\left(-Q_{1} F\right) e^{-A-t Q_{1} F} \\
& =-\int d \vec{y} \prod_{m>0} d z_{(m)}^{s} d z_{(m)}^{s *} d \zeta_{(m)}^{s} d \zeta_{(m)}^{s *} \mathcal{J} Q_{1}\left(F e^{-A-t Q_{1} F}\right)=0
\end{aligned}
$$


where in the last step we have used $Q_{1}$ invariance of the path integral measure. Thus $I(t)$ is independent of $t$, and has the same value in the limits $t \rightarrow 0$ and $t \rightarrow \infty$. Noting that in the $t \rightarrow 0$ limit $I(t)$ reduces to $I$, and using (3.6), (3.8) we get

$$
I=\lim _{t \rightarrow \infty} \int d \vec{y} \prod_{m>0} d z_{(m)}^{s} d z_{(m)}^{s *} d \zeta_{(m)}^{s} d \zeta_{(m)}^{s *} \mathcal{J} e^{-A-t \sum_{m>0} \sum_{s}\left[4 m z_{(m)}^{s *} z_{(m)}^{s}+\zeta_{(m)}^{s *} \zeta_{(m)}^{s}\right]}
$$

In the $t \rightarrow \infty$ limit the $z_{(m)}^{s}$ and $\zeta_{(m)}^{s}$ dependent terms inside the action $A$ are subleading. Thus up to an overall $t$ independent normalization constant 8 the $e^{-t \sum_{m>0} \sum_{s}\left[4 m z_{(m)}^{s *} z_{(m)}^{s}+\zeta_{(m)}^{s *} \zeta_{(m)}^{s}\right]}$ term in the $t \rightarrow \infty$ limit is equivalent to inserting in the path integral a factor of

$$
\prod_{m>0} \prod_{s} \delta\left(z_{(m)}^{s}\right) \delta\left(z_{(m)}^{s *}\right) \delta\left(\zeta_{(m)}^{s *}\right) \delta\left(\zeta_{(m)}^{s}\right)
$$

This shows that the path integral is localized in the subspace of $\left(\widehat{L}_{0}-J^{3}\right)$ invariant deformations parametrized by the coordinates $\vec{y}$. In particular it restricts integration over the orbits of $S U(1,1 \mid 2)$, generated by the action of (2.19) on any $\left(\widehat{L}_{0}-J^{3}\right)$ invariant configuration, to the subspace

$$
\xi=0, \quad \eta=0, \quad \theta_{\alpha \beta}=0 .
$$

More generally, since $\widehat{L}_{0}$ and $J^{3}$ generate translations along $\theta$ and $\phi$ directions of $A d S_{2} \times S^{2}$ respectively, restriction to $\widehat{L}_{0}-J^{3}$ invariant subspace amounts to restricting the path integral over field configurations which depend on $\theta$ and $\phi$ only through the combination $(\theta+\phi)$.

We can further localize the $\vec{y}$ integral onto $Q_{1}$-invariant subspace. Intuitively this can be understood by noting that unless $\vec{y}$ is invariant under $Q_{1}$, the orbit of $Q_{1}$ through a point $\vec{y}$ will give a vanishing contribution to the integral [15, 19]. Thus the contribution to the integral must come from the $Q_{1}$ invariant subspace of the $\left(\widehat{L}_{0}-J^{3}\right)=0$ subspace. Formally this can be established as follows. Let $\left(\left\{\vec{w}^{\alpha}\right\},\left\{\zeta^{a}\right\}\right)$ denote the bosonic and fermionic components of $\vec{y}$. Then we can write

$$
Q_{1} \zeta^{a}=f^{a}(\vec{w}, \vec{\zeta})
$$

for some functions $f^{a}$. We now insert into the path integral a term

$$
\exp \left[-t Q_{1} \sum_{a} \zeta^{a} f^{a}(\vec{w}, \vec{\zeta})\right]=\exp \left[-t \sum_{a} f^{a}(\vec{w}, \vec{\zeta}) f^{a}(\vec{w}, \vec{\zeta})\right]
$$

\footnotetext{
${ }^{8}$ This normalization constant can of course be absorbed into a redefinition of the measure $\mathcal{J}$. Alternatively, we could define $\zeta_{(m)}^{s}$ with a different normalization so that eqs. (3.3) take the form $Q_{1} z_{(m)}^{s}=$ $\alpha_{m} \zeta_{(m)}^{s}, \quad Q_{1} \zeta_{(m)}^{s}=4 m \alpha_{m}^{-1} z_{(m)}^{s}$ for some constant $\alpha_{m}$. By adjusting $\alpha_{m}$ we could ensure that the replacement of the $t$ dependent exponential factor by (3.12) does not require any additional normalization.
} 
Nilpotence of $Q_{1}$ and $Q_{1}$ invariance of the original action can be used to argue that the path integral is independent of $t$. Restriction of the path integral to the purely bosonic subspace $\zeta^{a}=0$ now has a factor $\exp \left\{-t \sum_{a} f^{a}(\vec{w}, \overrightarrow{0}) f^{a}(\vec{w}, \overrightarrow{0})\right\}$. Thus in the $t \rightarrow \infty$ limit the path integral is restricted to the subspace $f^{a}(\vec{w}, \overrightarrow{0})=0$ in the $\vec{\zeta}=0$ sector. This is precisely the $Q_{1}$ invariant subspace of purely bosonic configurations.

This establishes that in order to get a non-vanishing contribution from integration around a saddle point $\Phi$ it must be invariant under the group $H_{1}$ generated by $Q_{1}$ and $\widehat{L}_{0}$. Furthermore after taking into account appropriate measure factors we can express the path integral as integration over an $H_{1}$ invariant slice passing through $\Phi$.

One might wonder whether it is possible to argue that the path integral can be simultaneously localized into the subspace that is invariant under all the $Q_{i}$ 's 1.e. the subgroup $H_{0}$. An intuitive argument to this effect can be given as follows. We have chosen to define the path integral by first integrating over the orbits of $H_{1}$, then integrating over the orbits of the rest of the elements of $H_{0}$ and finally integrating over the rest of the variables in some order. Now since the bosonic subgroup of $H_{0}$ - generated by $\left(\widehat{L}_{0}-J^{3}\right)$ - is compact, integration along the orbit of this generator cannot produce a divergence. On the other hand integration over the orbit of any of the fermionic generators $Q_{i}$ will produce a zero. Thus unless the configuration is invariant under all the fermionic generators $Q_{i}$ of $H_{0}$, the contribution to the path integral from the orbits of $H_{0}$ through this configuration will vanish.

One however runs into problem in trying to construct a formal proof of this intuitive expectation. Naively one could proceed by first showing localization under $Q_{1}$ as we have described above and then adding further terms to the action to localize the integral into subspaces invariant under the other $Q_{i}$ 's. The problem with this approach is that generically the extra insertion $\exp \left(-t Q_{1} F\right)$ into the path integral in (3.6) may not maintain invariance under the other $Q_{i}$ 's. As a result the $Q_{i}$ 's are not symmetries of the extra factor given in (3.12). Similar problem arises for the factor (3.15). We believe that this is essentially a technical issue and the path integral really receives contribution only from the $H_{0}$ invariant configurations. However we shall take a conservative approach and use only the requirement of $H_{1}$ invariance in our subsequent analysis. In particular the analysis of $\$$, showing that the infinite set of fermionic and bosonic zero modes arising from the asymptotic symmetries of $A d S_{2}$ give a finite contribution to the path integral, will rely only on localization onto $\left(\widehat{L}_{0}-J^{3}\right)$ invariant subspace. In $\$ 5$ we shall describe freely acting orbifolds of the original $A d S_{2} \times S^{2}$ background which contribute to the path integral as new saddle points. Localization requires us to look for 
$H_{1}$ invariant orbifolds. However as we shall see, the requirement of $H_{1}$ invariance automatically forces us to have orbifolds invariant under the full $H_{0}$ subgroup of $S U(1,1 \mid 2)$.

\section{Integrating Over the Orbit of the Superconformal Cur- rent Algebra}

String theory on $A d S_{2} \times S^{2}$ space, describing the near horizon geometry of a BPS black hole, has an infinite group of asymptotic symmetries besides the global $S U(1,1 \mid 2)$ transformations which leave the $A d S_{2} \times S^{2}$ background invariant. These more general transformations do not leave the $A d S_{2} \times S^{2}$ background invariant but preserve the asymptotic condition on the various fields. Hence they can be used to generate new solutions from a given solution. As was shown in [3], the Euclidean action of the theory remains unchanged under these transformations even after taking into account the effect of the infrared cut-off. Thus they represent zero modes. In a non-supersymmetric theory where only bosonic zero modes are present, integration over these zero modes will generate an infinite factor in the partition function. Hence integration over these directions must be restricted by declaring the corresponding transformations as gauge transformations. However as was pointed out in [3], in a supersymmetric theory there is a possibility of cancellation between the bosonic and fermionic zero mode integrals yielding a finite result. We shall now demonstrate that this is indeed what happens.

The generators of the extended superconfomal algebra may be labelled as $\widetilde{L}_{n}, \widetilde{J}_{n}^{i}$ and $\widetilde{G}_{r}^{\alpha \beta}$ with $n \in \mathbb{Z}, r \in \mathbb{Z}+\frac{1}{2}, 1 \leq i \leq 3$ and $\alpha, \beta= \pm$. The generators of $s u(1,1 \mid 2)$ discussed in $\oiint_{2}$ are special cases of these generators with the identification

$$
\widehat{L}_{0}=\widetilde{L}_{0}, \quad \widehat{L}_{ \pm}=\widetilde{L}_{\mp 1}, \quad J^{i}=\widetilde{J}_{0}^{i}, \quad \widehat{G}_{ \pm}^{\alpha \beta}=\widetilde{G}_{\mp \frac{1}{2}}^{\alpha \beta} .
$$

For our analysis we shall not need the full superconformal current algebra, but only the commutators of the various generators with $\widehat{L}_{0}$ and $J^{3}$. They are given by

$$
\begin{aligned}
{\left[\widehat{L}_{0}, \widetilde{L}_{n}\right]=} & -n \widetilde{L}_{n}, \quad\left[\widehat{L}_{0}, \widetilde{J}_{n}^{i}\right]=-n \widetilde{J}_{n}^{i}, \quad\left[\widehat{L}_{0}, \widetilde{G}_{r}^{\alpha \beta}\right]=-r \widetilde{G}_{r}^{\alpha \beta} \\
{\left[J^{3}, \widetilde{L}_{n}\right]=} & {\left[J^{3}, \widetilde{J}_{n}^{3}\right]=0, \quad\left[J^{3}, \widetilde{J}_{n}^{ \pm}\right]= \pm \widetilde{J}_{n}^{ \pm}, \quad\left[J^{3}, \widetilde{G}_{r}^{\alpha \beta}\right]=\frac{1}{2} \beta \widetilde{G}_{r}^{\alpha \beta} } \\
& \widetilde{J}_{n}^{ \pm} \equiv \widetilde{J}_{n}^{1} \pm i \widetilde{J}_{n}^{2}
\end{aligned}
$$

This gives

$$
\left[\widehat{L}_{0}-J^{3}, \widetilde{L}_{n}\right]=-n \widetilde{L}_{n}, \quad\left[\widehat{L}_{0}-J^{3}, \widetilde{J}_{n}^{3}\right]=-n \widetilde{J}_{n}^{3}, \quad\left[\widehat{L}_{0}-J^{3}, \widetilde{J}_{n}^{ \pm}\right]=(-n \mp 1) \widetilde{J}_{n}^{ \pm}
$$




$$
\left[\widehat{L}_{0}-J^{3}, \widetilde{G}_{r}^{\alpha \beta}\right]=\left(-\frac{1}{2} \beta-r\right) \widetilde{G}_{r}^{\alpha \beta} .
$$

Consider now an $H_{1}$-invariant saddle point and analyze the contribution from the zero modes generated by the action of the superconformal algebra. First note that most of the modes generated by the superconforml algebra carry non-zero eigenvalues under $\widehat{L}_{0}-J^{3}$. They are part of the deformations labelled by $z_{(m)}^{s}$ and $\zeta_{(m)}^{s}$ in $\oint 3$ and are eliminated by the localization procedure described in $\$ 3$. Thus we only need to worry about deformations generated by $\widehat{L}_{0}-J^{3}$ invariant generators. Of these several are part of the global symmetry group $S U(1,1 \mid 2)$ and have already been taken into account in the analysis of \$3. From (4.3) we see that the only $\widehat{L}_{0}-J^{3}$ invariant generators which are not part of $S U(1,1 \mid 2)$ are $\widetilde{J}_{-1}^{+}$and $\widetilde{J}_{1}^{-}$. Since together with $\widetilde{J}_{0}^{3}=J^{3}$ they generate an $S U(2)$ group, the integration over these zero modes will give us a finite factor proportional to the volume of $S U(2)$. This shows that around an $H_{1}$-invariant saddle point, integration over the fermionic and bosonic zero modes generated by the full superconformal current algebra gives a finite result.

\section{Examples of $H_{1}$-invariant Saddle Points}

In this section we shall review the construction of a class of saddle points from orbifolds of the near horizon geometry of the black hole [2,3, 26] and verify their $H_{1}$-invariance. We shall focus on type IIB string theory on $K 3$ - the theory discussed in appendix $\mathrm{A}$ - and consider six dimensional geometries whose asymptotic form coincide with that of $S^{1} \times \widetilde{S}^{1} \times A d S_{2} \times S^{2}$ with background 3 -form fluxes 9 The simplest $H_{1}$-invariant saddle point is $S^{1} \times \widetilde{S}^{1} \times A d S_{2} \times S^{2}$ with background fluxes, given in (A.26):

$$
\begin{aligned}
d s^{2} & =v\left(d \eta^{2}+\sinh ^{2} \eta d \theta^{2}\right)+u\left(d \psi^{2}+\sin ^{2} \psi d \phi^{2}\right)+\frac{R^{2}}{\tau_{2}}\left|d x^{4}+\tau d x^{5}\right|^{2}, \\
G^{I} & =\frac{1}{8 \pi^{2}}\left[Q_{I} \sin \psi d x^{5} \wedge d \psi \wedge d \phi+P_{I} \sin \psi d x^{4} \wedge d \psi \wedge d \phi+\text { dual }\right], \\
V_{I}{ }^{i} & =\text { constant, } \quad V_{I}^{r}=\text { constant. }
\end{aligned}
$$

As discussed in appendix $\mathrm{A}$, this background is invariant under the full $S U(1,1 \mid 2)$ symmetry group. The classical contribution to the quantum entropy function from this saddle point is given by $\exp \left(S_{\text {wald }}\right)$ where $S_{\text {wald }}$ denotes the classical Wald entropy [7].

\footnotetext{
${ }^{9}$ Note $S^{1}$ and $\widetilde{S}^{1}$ are not factored metrically, 1.e. we allow the metric to have components which mix $S^{1}$ and $\widetilde{S}^{1}$ coordinates.
} 
We shall now construct other $H_{1}$ invariant saddle points with the same asymptotic behaviour as (5.1) by taking orbifold of the above background by some discrete $\mathbb{Z}_{s}$ group. Since $H_{1}$ is generated by $Q_{1}$ and $Q_{1}^{2}$, in order to preserve $H_{1}$ the $\mathbb{Z}_{s}$ action must commute with $Q_{1}$. Typically the generator of the $\mathbb{Z}_{s}$ transformation will involve an element of $S U(1,1 \mid 2)$ together with an internal symmetry transformation that commutes with $S U(1,1 \mid 2)$. Now one can see from the algebra (2.13) that the only bosonic generator of $s u(1,1 \mid 2)$ that commutes with $Q_{1}$ is $\left(\widehat{L}_{0}-J^{3}\right)$. Thus the part of the orbifold group generator that belongs to $S U(1,1 \mid 2)$ must be an element of the $U(1)$ subgroup generated by $\left(\widehat{L}_{0}-J^{3}\right)$. However since $\left(\widehat{L}_{0}-J^{3}\right)$ commutes with the $H_{0} \times U(1)$ subgroup of $S U(1,1 \mid 2)$ generated by $Q_{1}, \cdots Q_{4}$ and $\left(\widehat{L}_{0} \pm J^{3}\right)$, we see that any such saddle point will automatically also be invariant under this bigger subgroup of $S U(1,1 \mid 2)$. We shall now give some specific examples of such orbifolds.

It was shown in [27, 28] that with the help of a duality transformation we can bring the charge vector to the form

$$
(Q, P)=\left(\ell Q_{0}, P_{0}\right),
$$

for some integer $\ell$, representing a duality invariant combination of the charges [29]. Here $\left(Q_{0}, P_{0}\right)$ are primitive vectors of the charge lattice, satisfying

$$
\operatorname{gcd}\left(\left\{Q_{0 I} P_{0 J}-Q_{0 J} P_{0 I}\right\}\right)=1 .
$$

We now consider an orbifold of the background (5.1) by the $\mathbb{Z}_{s}$ transformation 3$]$

$$
\left(\theta, \phi, x^{5}\right) \rightarrow\left(\theta+\frac{2 \pi}{s}, \phi-\frac{2 \pi}{s}, x^{5}+\frac{2 \pi k}{s}\right), \quad k, s \in \mathbb{Z}, \quad \operatorname{gcd}(s, k)=1 .
$$

Since the circle parametrized by $x^{5}$ is non-contractible, this is a freely acting orbifold. At the origin $\eta=0$ of the $A d S_{2}$ space we have a non-contractible 3 -cycle spanned by $\left(x^{5}, \psi, \phi\right)$, with the identification $\left(x^{5}, \psi, \phi\right)=\left(x^{5}+2 \pi k / s, \psi, \phi-2 \pi / s\right)$. As a result of this identification the total flux of $G^{I}$ through this cycle is equal to $Q_{I} / s=(l / s) Q_{0 I}$. Since the flux quantization constraints require the fluxes through this new 3 -cycle to be integers, we see that this orbifold is an allowed configuration in string theory only when $\ell / s$ is an integer.

Since $\left(\widehat{L}_{0}-J^{3}\right)$ shifts $\theta$ and $\phi$ in opposite directions, the $\mathbb{Z}_{s}$ transformation described in (5.4) is generated by $\left(\widehat{L}_{0}-J^{3}\right)$ together with a shift along $x^{5}$. Since all the generators of $S U(1,1 \mid 2)$ are invariant under a shift along $x^{5}$, we see that the subgroup of $S U(1,1 \mid 2)$ that commutes with $\left(\widehat{L}_{0}-J^{3}\right)$ will be a symmetry of this orbifold. This is precisely the group $H_{0}$ together with the $U(1)$ subgroup generated by $\left(\widehat{L}_{0}+J^{3}\right)$. Indeed one can verify explicitly 
that the Killing spinors associated with the generators $G_{+}^{++}, G_{-}^{--}, G_{+}^{-+}$and $G_{-}^{+-}$described in (A.37) are invariant under the transformation (5.4).

It was shown in [3] that the orbifold described above has the correct asymptotic behaviour. For this we rename the coordinates $\left(\eta, \theta, \phi, x^{5}\right)$ appearing in (A.26) as $\left(\widetilde{\eta}, \widetilde{\theta}, \widetilde{\phi}, \widetilde{x}^{5}\right)$ and express the new configuration as

$$
\begin{aligned}
d s^{2} & =v\left(d \widetilde{\eta}^{2}+\sinh ^{2} \widetilde{\eta} d \widetilde{\theta}^{2}\right)+u\left(d \psi^{2}+\sin ^{2} \psi d \widetilde{\phi}^{2}\right)+\frac{R^{2}}{\tau_{2}}\left|d x^{4}+\tau d \widetilde{x}^{5}\right|^{2} \\
G^{I} & =\frac{1}{8 \pi^{2}}\left[Q_{I} \sin \psi d \widetilde{x}^{5} \wedge d \psi \wedge d \widetilde{\phi}+P_{I} \sin \psi d x^{4} \wedge d \psi \wedge d \widetilde{\phi}+\text { dual }\right] \\
\left(\widetilde{\theta}, \widetilde{\phi}, \widetilde{x}^{5}\right) & \equiv\left(\widetilde{\theta}+\frac{2 \pi}{s}, \widetilde{\phi}-\frac{2 \pi}{s}, \widetilde{x}^{5}+\frac{2 \pi k}{s}\right) \equiv\left(\widetilde{\theta}+2 \pi, \widetilde{\phi}, \widetilde{x}^{5}\right) \\
& \equiv\left(\widetilde{\theta}, \widetilde{\phi}+2 \pi, \widetilde{x}^{5}\right) \equiv\left(\widetilde{\theta}, \widetilde{\phi}, \widetilde{x}^{5}+2 \pi\right) .
\end{aligned}
$$

We now make the coordinate transformation:

$$
\theta=s \widetilde{\theta}, \quad \phi=\widetilde{\phi}+(1-s) \widetilde{\theta}, \quad x^{5}=\widetilde{x}^{5}-k \widetilde{\theta}, \quad \eta=\widetilde{\eta}-\ln s
$$

In these coordinates the background (5.5) takes the form

$$
\begin{aligned}
d s^{2}= & v\left(d \eta^{2}+\sinh ^{2} \eta\left(1+\frac{\left(1-s^{-2}\right) e^{-\eta}}{2 \sinh \eta}\right)^{2} d \theta^{2}\right)+u\left(d \psi^{2}+\sin ^{2} \psi\left(d \phi+d \theta-s^{-1} d \theta\right)^{2}\right) \\
& +\frac{R^{2}}{\tau_{2}}\left|d x^{4}+\tau\left(d x^{5}+k s^{-1} d \theta\right)\right|^{2} \\
G^{I}= & \frac{1}{8 \pi^{2}}\left[Q_{I} \sin \psi\left(d x^{5}+k s^{-1} d \theta\right) \wedge d \psi \wedge\left(d \phi+d \theta-s^{-1} d \theta\right)\right. \\
& \left.+P_{I} \sin \psi d x^{4} \wedge d \psi \wedge\left(d \phi+d \theta-s^{-1} d \theta\right)+\text { dual }\right] \\
& \left(\theta, \phi, x^{5}\right) \equiv\left(\theta+2 \pi, \phi, x^{5}\right) \equiv\left(\theta, \phi+2 \pi, x^{5}\right) \equiv\left(\theta, \phi, x^{5}+2 \pi\right) .
\end{aligned}
$$

Since the asymptotic region lies at large $\eta$, we see that this has the same asymptotic behaviour as the $S^{1} \times \widetilde{S}^{1} \times A d S_{2} \times S^{2}$ background described in (A.26). Note the presence of the $d \theta-$ $s^{-1} d \theta$ terms added to $d \phi$ and $k s^{-1} d \theta$ terms added to $d x^{5}$. From the point of view of the two dimensional theory living on $A d S_{2}$ these represent constant values of the gauge fields arising from the $5 \theta$ and $\phi \theta$ components of the metric. As discussed in detail in [1,2,3], in defining the path integral over $A d S_{2}$ we must integrate over these modes. Thus (5.7) is an allowed configuration over which the path integral should be performed. The classical contribution to the quantum entropy function from this saddle point is given by $\exp \left(S_{\text {wald }} / s\right)$ [3]. These match 
with the asymptotic behaviour of specific extra terms in the microscopic formula which appear when the integer $\ell$ introduced in (5.2) is larger than 1 .

Starting with the Killing spinors given in (A.37) with $(\theta, \phi, \eta)$ replaced by $(\widetilde{\theta}, \widetilde{\phi}, \widetilde{\eta})$, and then using the coordinate transformations given in (5.6) one can verify that the Killing spinors corresponding to $G_{+}^{++}, G_{-}^{--}, G_{+}^{-+}$and $G_{-}^{+-}$are given by the same expressions as in (A.37) with $\eta$ replaced by $\eta+\ln s$. From the structure of (A.37) it can be seen that for large $\eta$ the replacement of $\eta$ by $\eta+\ln s$ multiplies the Killing spinors by an overall factor of $\sqrt{s}$. This is just an overall normalization constant and can be removed. Thus we see that asymptotically the Killing spinors of this new saddle point coincide with those of the background (5.1). The regularity of the Killing spinors at the origin follows from the fact that the new saddle point is obtained as a freely acting orbifold of (5.1) and that in the parent theory the Killing spinors were regular everywhere.

Finally we can consider another class of orbifolds for which $k$ appearing in (5.4) vanishes, or more generally, has a common factor with $s$. The orbifold group still commutes with $H_{0}$ and hence we expect $H_{0}$ to be a symmetry of this orbifold. However in this case the orbifold action has fixed points and we no longer have a freely acting orbifold. Let us consider the $k=0$ case for definiteness [2]. The points $(\eta=0 ; \psi=0, \pi)$ are fixed points of this orbifold group, and the 3 -cycles spanned by $\left(x^{5}, \psi, \phi\right)$ and $\left(x^{4}, \psi, \phi\right)$ at $\eta=0$ now pass through these fixed points. The fluxes through these three cycles from regions outside the fixed points are given by $Q_{I} / s$ and $P_{I} / s$ respectively. However flux quantization rule does not put any constraints on the charge vectors $Q_{I}$ and $P_{I}$. Instead it requires that there must be additional flux at the fixed points which make the total flux through these 3-cycles satisfy the correct quantization rules 10 As was argued in [2], the contribution to the partition function from these saddle points is given by $\exp \left(S_{\text {wald }} / s\right)$ if we ignore the contribution from the fixed points. Furthermore the contribution from the fixed points add at most constants of order unity to $S_{\text {wald }} / s$ whereas $S_{\text {wald }}$ grows quadratically with the charges carried by the black hole. Thus for large charges the contribution from the fixed points to the exponent is subleading.

In a dual description of these theories in $M$-theory the near horizon geometry of these black holes can have an extra circle that combines with the $A d S_{2}$ to give a locally $A d S_{3}$ space. In this case one can get freely acting $\mathbb{Z}_{s}$ orbifolds by accompanying the orbifold action by a translation along this extra circle [26], without imposing any additional arithmetic condition on the charges of the type $\ell / s \in \mathbb{Z}$. This could provide a possible way to analyze the orbifolds

\footnotetext{
${ }^{10}$ Such fluxes have been considered before in $[30$ in a different context.
} 
with fixed points in the type IIB description.

\section{Comments}

In this paper we have used the localization procedure to classify the saddle points which will contribute to string theory path integral over the near horizon geometry of extremal BPS black holes. This path integral is required for the computation of quantum entropy function, which appears in the macroscopic computation of the entropy of extremal black holes via $A d S_{2} / C F T_{1}$ correspondence [1].

We hope that the same localization techniques will also simplify the computation of the path integral around each of the saddle points, e.g. by reducing the path integral over the fields to a finite dimensional integral. In particular for quarter BPS black holes in type IIB string theory on $K 3 \times T^{2}$ if the contribution to the path integral from some of the saddle points can be expressed as finite dimensional integrals, they can then be compared with the corresponding microscopic results derived in [31, 32, 33, 34, 35, 36, 37, 38, providing us with a precision test of the $A d S_{2} / C F T_{1}$ correspondence. The formulation of string theory on $A d S_{2} \times S^{2}$ described in 39] could also be a useful tool in this venture.

Finally we note that drawing inspiration from [40] a recent paper [25] expressed the expectation value of circular 't Hooft - Wilson loop operators in an $\mathcal{N}=4$ supersymmetric gauge theory as a path integral over the field theory on $A d S_{2} \times S^{2}$ background. Except for the replacement of the string theory by $\mathcal{N}=4$ supersymmetric Yang-Mills theory, this path integral is identical to what appears in the definition of the quantum entropy function. Thus we expect that any method (like the one in the present paper) developed for the study of quantum entropy function is likely to be useful for the study of the 't Hooft - Wilson loop operators in $\mathcal{N}=4$ super-Yang-Mills theory. Similarly any method developed for computing 't Hooft - Wilson loop operators in $\mathcal{N}=4$ super-Yang Mills theory (like the one developed in [25]) may be useful for the computation of quantum entropy function in string theory. It will also be useful to explore whether the correspondence between the 't Hooft - Wilson loop and the quantum entropy function is just a mathematical coincidence or whether there is some deeper physical reason behind it.

Acknowledgement: We would like to thank Sayantani Bhattacharyya, Chethan Gowdigere, Dileep Jatkar and Yogesh Srivastava for useful discussions. 


\section{A Killing Spinors in Six Dimensional Supergravity on $S^{1} \times \widetilde{S}^{1} \times A d S_{2} \times S^{2}$}

In this appendix we shall analyze the Killing spinors in six dimensional $\mathcal{N}=4$ chiral supergravity compactified on $S^{1} \times \widetilde{S}^{1} \times A d S_{2} \times S^{2}$. This theory is dual to M-theory on $K 3 \times T^{3} \times A d S_{2} \times S^{2}$, for which the Killing spinor equations have been analyzed in [24]. Thus we could try to recover our answer by dualizing the results of [24]. We shall however analyze the Killing spinor equations directly in the six dimensional chiral supergravity in the presence of arbitrary background fluxes. This will make the duality covariance of the equations manifest.

We begin with the six dimensional supergravity theory obtained by dimensional reduction of type IIB supergravity on $K 3$ [41,42]. We shall follow the conventions of [43]. The bosonic fields in the theory are the metric $g_{M N}$, matrix valued scalar fields $V_{I}{ }^{i}, V_{I}{ }^{r}(1 \leq i \leq 5$, $6 \leq r \leq 26)$ satisfying

$$
V L V^{T}=L, \quad L=\operatorname{diag}\left(+{ }^{5},-{ }^{21}\right),
$$

and 2-form fields $B_{M N}^{I}(1 \leq I \leq 26)$ with field strengths $G^{I}=d B^{I}$ satisfying the following self duality constraint:

$$
H_{M N P}^{i}=\frac{1}{3 !} e_{M N P Q R S} H^{i Q R S}, \quad H_{M N P}^{r}=-\frac{1}{3 !} e_{M N P Q R S} H^{r Q R S},
$$

where

$$
H_{M N P}^{i}=G_{M N P}^{I} V_{I}^{i}, \quad H_{M N P}^{r}=G_{M N P}^{I} V_{I}^{r} .
$$

$e_{M N P Q R S}$ is a six form defined via

$$
e^{M N P Q R S}=|\operatorname{det} g|^{-1 / 2} \epsilon^{M N P Q R S},
$$

$\epsilon$ being the totally antisymmetric symbol. We shall label the time coordinate by $t$ and the space-coordinates by $\left(x^{4}, x^{5}, \eta, \psi, \phi\right)$ and choose the convention

$$
\epsilon^{t 45 \eta \psi \phi}=1 .
$$

Indices of $e$ are raised and lowered by the metric $g_{M N}$. Not all components of $V$ describe physical degrees of freedom since there is an identification

$$
V \equiv V O
$$

where $O$ is an $S O(5) \times S O(21)$ matrix acting on the first five and the last twenty one indices respectively. 
In the sector where the bosonic fields are taken to be space-time independent constants, the equations of motion take the form

$$
\begin{gathered}
R_{M N}=H_{M P Q}^{i} H_{N}^{i P Q}+H_{M P Q}^{r} H_{N}^{r P Q} \\
H_{M N P}^{i} H^{r M N P}=0,
\end{gathered}
$$

where $R_{M N}$ is the Ricci tensor defined in the sign convention in which on the sphere the Ricci scalar $g^{M N} R_{M N}$ is positive. We now look for a solution in this theory of the form

$$
\begin{aligned}
d s^{2} & =v\left(d \eta^{2}-\sinh ^{2} \eta d t^{2}\right)+u\left(d \psi^{2}+\sin ^{2} \psi d \phi^{2}\right)+\frac{R^{2}}{\tau_{2}}\left|d x^{4}+\tau d x^{5}\right|^{2}, \\
G^{I} & \equiv \frac{1}{3 !} G_{M N P}^{I} d x^{M} \wedge d x^{N} \wedge d x^{P} \\
& =\frac{1}{8 \pi^{2}}\left[Q_{I} \sin \psi d x^{5} \wedge d \psi \wedge d \phi+P_{I} \sin \psi d x^{4} \wedge d \psi \wedge d \phi+\text { dual }\right], \\
V_{I}{ }^{i} & =\text { constant }, \quad V_{I}{ }^{r}=\text { constant. }
\end{aligned}
$$

Here 'dual' denotes the dual 3-form required to make $G^{I}$ satisfy the self-duality constraint given in (A.2) $, v, u, R$ are real constants and $\tau=\tau_{1}+i \tau_{2}$ is a complex constant. ( $\left.\eta, t\right)$ label an $A d S_{2}$ space, $(\psi, \phi)$ label a 2-sphere and $x^{4}, x^{5}$ label coordinates along $\widetilde{S}^{1}$ and $S^{1}$ respectively, each taken to have period $2 \pi . Q_{I}$ and $P_{I}$ denote the fluxes through the 3 -cycles $S^{1} \times S^{2}$ and $\widetilde{S}^{1} \times S^{2}$ respectively, and are related to the integer charges carried by the black hole whose near horizon geometry is described by (A.8). In order to solve (A.7) we note that given any charge vectors $(Q, P)$ satisfying

$$
Q^{2}>0, \quad P^{2}>0, \quad Q^{2} P^{2}>(Q \cdot P)^{2},
$$

where

$$
Q^{2}=Q^{T} L Q, \quad P^{2}=P^{T} L P, \quad Q \cdot P=Q^{T} L P,
$$

we can always find a matrix $S$ satisfying $S L S^{T}=L$ such that

$$
Q=S Q_{0}, \quad P=S P_{0}
$$

where

$$
Q_{0}=\left(\begin{array}{c}
Q \cdot P / \sqrt{P^{2}} \\
\sqrt{Q^{2} P^{2}-(Q \cdot P)^{2}} / \sqrt{P^{2}} \\
0 \\
\cdot \\
\cdot
\end{array}\right), \quad P_{0}=\left(\begin{array}{c}
\sqrt{P^{2}} \\
0 \\
0 \\
\cdot \\
\cdot
\end{array}\right) \text {. }
$$


In that case eqs. (A.7) is solved by (A.8) for the choice

$$
\begin{aligned}
& V=\left(S^{T}\right)^{-1}, \quad \tau_{1}=Q \cdot P / P^{2}, \quad \tau_{2}=\sqrt{Q^{2} P^{2}-(Q \cdot P)^{2}} / P^{2}, \\
& v=u=\frac{1}{16 \pi^{4} R^{2}} \sqrt{Q^{2} P^{2}-(Q \cdot P)^{2}} .
\end{aligned}
$$

Using eq.(A.3) this gives

$$
H^{i}=\frac{1}{8 \pi^{2}}\left[Q_{0}^{i} \sin \psi d x^{5} \wedge d \psi \wedge d \phi+P_{0}^{i} \sin \psi d x^{4} \wedge d \psi \wedge d \phi+\text { dual }\right], \quad H^{r}=0 .
$$

Note that $R$ is arbitrary. Furthermore $S$ defined through (A.11) is ambiguous up to an $S O(3,21)$ transformation from the right acting on the last 24 elements. Thus $V$ given in (A.13) is determined only up to an $S O(3,21)$ multiplication from the right. Due to the identification (A.6) only an $S O(3,21) / S O(3) \times S O(21)$ family of these describe physically inequivalent configurations. These parameters which are left undetermined by the equations of motion describe flat directions of the entropy function.

The fermion fields in this theory consist of a set of gravitini $\psi_{M}$ and a set of spin $1 / 2$ fermions $\chi^{r} \cdot \chi^{r}$ transforms as 21 of $S O(21), \mathbf{4}$ of $S O(5)$ and a right chiral spinor of $S O(5,1)$ where $S O(5,1)$ denotes the tangent space Lorentz group, $S O(21)$ is the internal symmetry group acting on the index $r$, and $S O(5)$ is the internal symmetry group acting on the index $i$. In what follows we shall suppress all the $S O(5) \times S O(5,1)$ spinor indices. For each $M$, $\psi_{M}$ transforms as 4 of $S O(5)$ and a left-chiral spinor of $S O(5,1)$. Finally the supersymmetry transformation parameter $\epsilon$ transforms as a $\mathbf{4}$ of $S O(5)$ and a left chiral spinor of $S O(5,1)$. Let us denote the vielbeins by $e_{M}^{A}$ with $A$ labelling an $S O(5,1)$ tangent space index, the $\operatorname{SO}(5,1)$ gamma matrices by $\widetilde{\Gamma}^{A}$ and the $\mathrm{SO}(5)$ gamma matrices by $\widehat{\Gamma}^{i}$. We shall also use the symbol $\Gamma^{M}$ to denote the $S O(5,1)$ gamma matrices in the coordinate basis, 1.e. we have

$$
\widetilde{\Gamma}^{A}=e_{M}^{A} \Gamma^{M}
$$

Then the $S O(5,1)$ chirality conditions on various spinors may be described as

$$
\begin{aligned}
& \left(\Gamma^{Q R S}-\frac{1}{3 !} e^{M N P Q R S} \Gamma_{M N P}\right) \chi^{r}=0, \\
& \left(\Gamma^{Q R S}+\frac{1}{3 !} e^{M N P Q R S} \Gamma_{M N P}\right) \psi_{K}=0, \\
& \left(\Gamma^{Q R S}+\frac{1}{3 !} e^{M N P Q R S} \Gamma_{M N P}\right) \epsilon=0
\end{aligned}
$$


where

$$
\Gamma^{M_{1} \cdots M_{k}}=\frac{1}{k !}\left(\Gamma^{M_{1}} \cdots \Gamma^{M_{k}}+\text { permutations with sign }\right) .
$$

Besides this all the spinors $\psi_{M}, \chi^{r}$ and $\epsilon$ satisfy the symplectic Majorana condition, e.g. we have

$$
\bar{\epsilon}=\epsilon^{T} C \Omega,
$$

where $\Omega$ is the $\mathrm{SO}(5)$ charge conjugation matrix acting on the $S O(5)$ spinor index and $C$ is the $\mathrm{SO}(5,1)$ charge conjugation matrix acting on the $\mathrm{SO}(5,1)$ spinor index. The supersymmetry transformation laws of various fields take the form

$$
\begin{aligned}
& \delta e_{M}^{A}=\bar{\epsilon} \widetilde{\Gamma}^{A} \psi_{M} \\
& \delta \psi_{M}=D_{M} \epsilon-\frac{1}{4} H_{M N P}^{i} \Gamma^{N P} \widehat{\Gamma}^{i} \epsilon, \quad D_{M} \epsilon \equiv \partial_{M} \epsilon+\frac{1}{4} \omega_{M}^{A B} \widetilde{\Gamma}_{A B} \epsilon-\frac{1}{4} Q_{M}^{i j} \widehat{\Gamma}^{i j} \epsilon, \\
& \omega_{M}^{A B} \equiv-g^{N P} e_{N}^{B} \partial_{M} e_{P}^{A}+e_{N}^{A} e_{P}^{B} g^{P Q} \Gamma_{Q M}^{N}, \quad \Gamma_{N P}^{M} \equiv \frac{1}{2} g^{M R}\left(\partial_{N} g_{P R}+\partial_{P} g_{N R}-\partial_{R} g_{N P}\right), \\
& \delta B_{M N}^{I}=-V^{I i} \bar{\epsilon} \Gamma_{[M} \widehat{\Gamma}^{i} \psi_{N]}+\frac{1}{2} V^{I r} \bar{\epsilon} \Gamma_{M N} \chi^{r}, \\
& \delta \chi^{r}=\frac{1}{\sqrt{2}} \Gamma^{M} P_{M}^{i r} \widehat{\Gamma}^{i} \epsilon+\frac{1}{12} \Gamma^{M N P} H_{M N P}^{r} \epsilon, \\
& \delta V_{I}^{i}=\bar{\epsilon} \widehat{\Gamma}^{i} \chi^{r} V_{I}^{r} \\
& \delta V_{I}^{r}=\bar{\epsilon} \widehat{\Gamma}^{i} \chi^{r} V_{I}^{i},
\end{aligned}
$$

where the index $I$ is raised and lowered by the matrix $L$ and

$$
P_{M}^{i r}=\frac{1}{\sqrt{2}} \partial_{M} V_{I}{ }^{i}\left(V^{-1}\right)_{r}{ }^{I}, \quad Q_{M}^{i j}=\partial_{M} V_{I}{ }^{i}\left(V^{-1}\right)_{j}{ }^{I} .
$$

Thus the Killing spinor equations, obtained by setting the variation of $\chi^{r}$ and $\psi_{M}$ to zero, are given by

$$
\begin{aligned}
& D_{M} \epsilon-\frac{1}{4} H_{M N P}^{i} \Gamma^{N P} \widehat{\Gamma}^{i} \epsilon=0, \\
& \frac{1}{\sqrt{2}} \Gamma^{M} P_{M}^{i r} \widehat{\Gamma}^{i} \epsilon+\frac{1}{12} \Gamma^{M N P} H_{M N P}^{r} \epsilon=0 .
\end{aligned}
$$

We shall try to solve these equations in the background (A.8), (A.13). The analysis simplifies if we note that in this background

$$
P_{M}^{i r}=0, \quad Q_{M}^{i j}=0, \quad H_{M N P}^{r}=0 .
$$


Thus the second set of equations in (A.21) are satisfied automatically. The first set of equations can be split into two sets by taking $M=(4,5)$ and $M=(\eta, t, \psi, \phi)$ :

$$
\begin{aligned}
& H_{a \mu \nu}^{i} \Gamma^{\mu \nu} \widehat{\Gamma}^{i} \epsilon=0, \quad a=4,5, \quad \mu, \nu=\eta, t, \psi, \phi, \\
& D_{\mu} \epsilon+\frac{1}{2} H_{a \mu \nu}^{i} \Gamma^{a \nu} \widehat{\Gamma}^{i} \epsilon=0 .
\end{aligned}
$$

Since we shall eventually be interested in finding the Killing spinors in the euclidean theory, we shall now make a euclidean continuation of the theory. This is done by making the replacement

$$
t \rightarrow-i \theta
$$

and replacing (A.4), (A.5) by

$$
e^{M N P Q R S}=i|\operatorname{det} g|^{-1 / 2} \epsilon^{M N P Q R S}, \quad \epsilon^{\theta 45 \eta \psi \phi}=1 .
$$

This will guarantee that a solution obtained by euclidean rotation of a Minkowski solution will satisfy the self-duality conditions (A.2) with $e_{M N P Q R S}$ defined via (A.25). Furthermore the chirality projection rules (A.16), the supersymmetry transformation rules (A.19) and the killing spinor equations (A.21) all remain unchanged as long as we use the new definition (A.25). Finally since the 4 representation of $S O(6)$ is different from its conjugate representation $\overline{\mathbf{4}}$, we can no longer impose the symplectic Majorana condition on the spinors. However we shall now take (A.18) as the definition of $\bar{\epsilon}$ appearing in the supersymmetry transformation laws. Equivalently, we could first replace $\bar{\epsilon}$ in the supersymmetry transformation laws in terms of $\epsilon$ using (A.18), and then make the Euclidean continuation. The charge conjugation matrices $C$ and $\Omega$ have to be chosen so that $\epsilon_{1}^{T} C \Omega \widetilde{\Gamma}^{A} \epsilon_{2}$ and $\epsilon_{1}^{T} C \Omega \widehat{\Gamma}^{i} \epsilon_{2}$ transform as $S O(6)$ vectors and $S O(5)$ vectors respectively for arbitrary $\epsilon_{1}$ and $\epsilon_{2}$.

Under the euclidean continuation the solution given in (A.8) takes the form:

$$
\begin{aligned}
d s^{2} & =v\left(d \eta^{2}+\sinh ^{2} \eta d \theta^{2}\right)+u\left(d \psi^{2}+\sin ^{2} \psi d \phi^{2}\right)+\frac{R^{2}}{\tau_{2}}\left|d x^{4}+\tau d x^{5}\right|^{2}, \\
G^{I} & =\frac{1}{8 \pi^{2}}\left[Q_{I} \sin \psi d x^{5} \wedge d \psi \wedge d \phi+P_{I} \sin \psi d x^{4} \wedge d \psi \wedge d \phi+\text { dual }\right], \\
V_{I}^{i} & =\text { constant, } \quad V_{I}^{r}=\text { constant }, \\
H^{i} & =\frac{1}{8 \pi^{2}}\left[Q_{0}^{i} \sin \psi d x^{5} \wedge d \psi \wedge d \phi+P_{0}^{i} \sin \psi d x^{4} \wedge d \psi \wedge d \phi+\text { dual }\right], \quad H^{r}=0,
\end{aligned}
$$


with the various parameters determined from (A.13). The equations (A.23) take the form

$$
\begin{aligned}
& H_{a \mu \nu}^{i} \Gamma^{\mu \nu} \widehat{\Gamma}^{i} \epsilon=0, \quad a=4,5, \quad \mu, \nu=\eta, \theta, \psi, \phi, \\
& D_{\mu} \epsilon+\frac{1}{2} H_{a \mu \nu}^{i} \Gamma^{a \nu} \widehat{\Gamma}^{i} \epsilon=0 .
\end{aligned}
$$

Using the self-duality constraints (A.2), the chirality constraints (A.16), the explicit form of the solutions given in (A.13), and (A.12), the first set of equations in (A.27) takes the simple form

$$
\widehat{\Gamma}^{1} \epsilon=\Gamma_{45}\left(\operatorname{det} g^{(45)}\right)^{-1 / 2} \widehat{\Gamma}^{2} \epsilon,
$$

where $g^{(45)}$ denotes the metric on $S^{1} \times \widetilde{S}^{1}$. We shall now use (A.28) to simplify the second set of equations in (A.27). For this we need to choose the vielbeins $e_{M}^{A}$ consistent with the background (A.26). We define $e^{A} \equiv e_{M}^{A} d x^{M}$ and take

$$
\begin{aligned}
& e^{0}=\sqrt{v} \sinh \eta d \theta, \quad e^{1}=\sqrt{v} d \eta, \quad e^{2}=\sqrt{u} \sin \psi d \phi, \quad e^{3}=\sqrt{u} d \psi, \\
& e^{4}=\frac{R}{\sqrt{\tau}_{2}}\left(d x^{4}+\tau_{1} d x^{5}\right), \quad e^{5}=R \sqrt{\tau_{2}} d x^{5} .
\end{aligned}
$$

We also denote by $x^{m}$ for $m=2,3$ the coordinates $(\phi, \psi)$ along $S^{2}$ and by $x^{\alpha}$ for $\alpha=0,1$ the coordinates $(\theta, \eta)$ along $A d S^{2}$. In that case the second set of equations in (A.27) are given by

$$
\begin{aligned}
& D_{m} \epsilon-\frac{1}{2} \sqrt{u} \varepsilon_{m n}^{S^{2}} \Gamma^{n} \widetilde{\Gamma}^{4} \widehat{\Gamma}^{1} \epsilon=0, \\
& D_{\alpha} \epsilon+\frac{i}{2} \sqrt{v} \varepsilon_{\alpha \beta}^{A d S_{2}} \Gamma^{\beta} \widetilde{\Gamma}^{4} \widehat{\Gamma}^{2} \epsilon=0,
\end{aligned}
$$

where $\widetilde{\Gamma}^{A}$ have been defined in (A.15), and

$$
\varepsilon_{m n}^{S^{2}} d x^{m} \wedge d x^{n}=\sin \psi d \psi \wedge d \phi, \quad \varepsilon_{\alpha \beta}^{A d S_{2}} d x^{\alpha} \wedge d x^{\beta}=\sinh \eta d \eta \wedge d \theta
$$

We can analyze these equations by choosing the following representation of the gamma matrices:

$$
\begin{aligned}
& \widetilde{\Gamma}^{0}=\sigma_{1} \otimes I \otimes I \otimes I \otimes I, \quad \widetilde{\Gamma}^{1}=\sigma_{2} \otimes I \otimes I \otimes I \otimes I, \quad \widetilde{\Gamma}^{2}=\sigma_{3} \otimes \sigma_{1} \otimes I \otimes I \otimes I \\
& \widetilde{\Gamma}^{3}=\sigma_{3} \otimes \sigma_{2} \otimes I \otimes I \otimes I, \quad \widetilde{\Gamma}^{4}=\sigma_{3} \otimes \sigma_{3} \otimes \sigma_{1} \otimes I \otimes I, \quad \widetilde{\Gamma}^{5}=\sigma_{3} \otimes \sigma_{3} \otimes \sigma_{2} \otimes I \otimes I \\
& \widehat{\Gamma}^{1}=I \otimes I \otimes I \otimes \sigma_{1} \otimes I, \quad \widehat{\Gamma}^{2}=I \otimes I \otimes I \otimes \sigma_{2} \otimes I, \quad \widehat{\Gamma}^{3}=I \otimes I \otimes I \otimes \sigma_{3} \otimes \sigma_{1} \\
& \widehat{\Gamma}^{4}=I \otimes I \otimes I \otimes \sigma_{3} \otimes \sigma_{2}, \quad \widehat{\Gamma}^{5}=I \otimes I \otimes I \otimes \sigma_{3} \otimes \sigma_{3},
\end{aligned}
$$


where the $\sigma_{i}$ are Pauli matrices and $I$ is the $2 \times 2$ identity matrix. In this basis the $S O(6)$ charge conjugation matrix $C$ and the $S O(5)$ charge conjugation matrix $\Omega$ have the form:

$$
C=i \sigma_{1} \otimes \sigma_{2} \otimes \sigma_{1} \otimes I \otimes I, \quad \Omega=I \otimes I \otimes I \otimes \sigma_{1} \otimes \sigma_{2},
$$

so that $C$ and $\Omega$ satisfy respectively the conditions for $S O(6)$ and $S O(5)$ invariance 11

$$
\left(C \widetilde{\Gamma}^{A}\right)^{T}=-C \widetilde{\Gamma}^{A}, \quad\left(\Omega \widehat{\Gamma}^{i}\right)^{T}=-\Omega \widehat{\Gamma}^{i}
$$

for all $A$ and $i$. We now note that the chirality condition (A.16) and the Killing spinor condition (A.28) leads to the constraints:

$$
\left(\sigma_{3} \otimes \sigma_{3} \otimes \sigma_{3} \otimes I \otimes I\right) \epsilon=\epsilon, \quad\left(I \otimes I \otimes \sigma_{3} \otimes \sigma_{3} \otimes I\right) \epsilon=-\epsilon .
$$

Due to these constraints we can parametrize $\epsilon$ by eight complex parameters $\left(\left\{A_{i}\right\},\left\{B_{i}\right\}\right)$ :

$$
\begin{aligned}
\epsilon= & \left(\begin{array}{l}
1 \\
0
\end{array}\right) \otimes\left(\begin{array}{l}
1 \\
0
\end{array}\right) \otimes\left(\begin{array}{l}
1 \\
0
\end{array}\right) \otimes\left(\begin{array}{l}
0 \\
1
\end{array}\right) \otimes\left(\begin{array}{l}
A_{1} \\
B_{1}
\end{array}\right)+\left(\begin{array}{l}
0 \\
1
\end{array}\right) \otimes\left(\begin{array}{l}
1 \\
0
\end{array}\right) \otimes\left(\begin{array}{l}
0 \\
1
\end{array}\right) \otimes\left(\begin{array}{l}
1 \\
0
\end{array}\right) \otimes\left(\begin{array}{l}
A_{2} \\
B_{2}
\end{array}\right) \\
& +\left(\begin{array}{l}
1 \\
0
\end{array}\right) \otimes\left(\begin{array}{l}
0 \\
1
\end{array}\right) \otimes\left(\begin{array}{l}
0 \\
1
\end{array}\right) \otimes\left(\begin{array}{l}
1 \\
0
\end{array}\right) \otimes\left(\begin{array}{l}
A_{3} \\
B_{3}
\end{array}\right)+\left(\begin{array}{l}
0 \\
1
\end{array}\right) \otimes\left(\begin{array}{l}
0 \\
1
\end{array}\right) \otimes\left(\begin{array}{l}
1 \\
0
\end{array}\right) \otimes\left(\begin{array}{l}
0 \\
1
\end{array}\right) \otimes\left(\begin{array}{l}
A_{4} \\
B_{4}
\end{array}\right) .
\end{aligned}
$$

Further simplification occurs due to the fact that eqs. A.30) do not mix the $A_{i}$ 's with $B_{i}$ 's and in fact remain invariant under the replacement $A_{i} \leftrightarrow B_{i}$. Thus we need to solve the Killing spinor equations in the four dimensional subspace parametrized by the $A_{i}$ 's (or $B_{i}$ 's). We get eight solutions $\zeta_{\gamma}^{\alpha \beta}(\alpha, \beta, \gamma= \pm)$. We shall first write down the solutions for $\zeta_{\gamma}^{+\beta}$. All of these solutions have $B_{i}=0$ and the $A_{i}$ 's given by:

$$
\begin{aligned}
& \zeta_{+}^{++}:\left(\begin{array}{c}
A_{1} \\
A_{2} \\
A_{3} \\
A_{4}
\end{array}\right)=2 v^{1 / 4} e^{i(\theta+\phi) / 2}\left(\begin{array}{c}
\sin \frac{\psi}{2} \sinh \frac{\eta}{2} \\
-\sin \frac{\psi}{2} \cosh \frac{\eta}{2} \\
-\cos \frac{\psi}{2} \sinh \frac{\eta}{2} \\
\cos \frac{\psi}{2} \cosh \frac{\eta}{2}
\end{array}\right), \\
& \zeta_{+}^{+-}:\left(\begin{array}{c}
A_{1} \\
A_{2} \\
A_{3} \\
A_{4}
\end{array}\right)=2 v^{1 / 4} e^{i(\theta-\phi) / 2}\left(\begin{array}{c}
-\cos \frac{\psi}{2} \sinh \frac{\eta}{2} \\
\cos \frac{\psi}{2} \cosh \frac{\eta}{2} \\
-\sin \frac{\psi}{2} \sinh \frac{\eta}{2} \\
\sin \frac{\psi}{2} \cosh \frac{\eta}{2}
\end{array}\right),
\end{aligned}
$$

\footnotetext{
${ }^{11}$ Note that A.34 does not fix the overall phases of $C$ and $\Omega$. We have chosen them according to our convenience.
} 


$$
\begin{aligned}
\zeta_{-}^{++}:\left(\begin{array}{l}
A_{1} \\
A_{2} \\
A_{3} \\
A_{4}
\end{array}\right) & =2 v^{1 / 4} e^{-i(\theta-\phi) / 2}\left(\begin{array}{c}
-\sin \frac{\psi}{2} \cosh \frac{\eta}{2} \\
\sin \frac{\psi}{2} \sinh \frac{\eta}{2} \\
\cos \frac{\psi}{2} \cosh \frac{\eta}{2} \\
-\cos \frac{\psi}{2} \sinh \frac{\eta}{2}
\end{array}\right), \\
\zeta_{-}^{+-}:\left(\begin{array}{l}
A_{1} \\
A_{2} \\
A_{3} \\
A_{4}
\end{array}\right) & =2 v^{1 / 4} e^{-i(\theta+\phi) / 2}\left(\begin{array}{c}
\cos \frac{\psi}{2} \cosh \frac{\eta}{2} \\
-\cos \frac{\psi}{2} \sinh \frac{\eta}{2} \\
\sin \frac{\psi}{2} \cosh \frac{\eta}{2} \\
-\sin \frac{\psi}{2} \sinh \frac{\eta}{2}
\end{array}\right) .
\end{aligned}
$$

The solutions for $\zeta_{\gamma}^{-\beta}$ are obtained by replacing the $A_{i}$ 's by $B_{i}$ 's and vice versa. The normalization factor $2 v^{1 / 4}$ has been included for convenience.

To check the regularity of the Killing spinors at the origin $\eta=0$ and / or $\psi=0, \pi$, we need to express the $A d S_{2} \times S^{2}$ metric in the $(z, w)$ coordinates as in (2.8) and choose the vielbeins as

$$
\begin{gathered}
\hat{e}^{0}=\frac{2 \sqrt{v}}{1-\bar{w} w} d w_{I}, \quad \hat{e}^{1}=\frac{2 \sqrt{v}}{1-\bar{w} w} d w_{R}, \quad \hat{e}^{2}=\frac{2 \sqrt{u}}{1+\bar{z} z} d z_{I}, \quad \hat{e}^{3}=\frac{2 \sqrt{u}}{1+\bar{z} z} d z_{R}, \\
w_{R}+i w_{I} \equiv w, \quad z_{R}+i z_{I} \equiv z .
\end{gathered}
$$

Since these vielbeins are regular at $w=0$ and / or $z=0$, the Killing spinors will be regular at these points if they are free from any singularity in this frame. Now using (A.29) we get

$$
\begin{array}{ll}
\hat{e}^{0}=\cos \theta e^{0}+\sin \theta e^{1}, & \hat{e}^{1}=-\sin \theta e^{0}+\cos \theta e^{1}, \\
\hat{e}^{2}=\cos \phi e^{2}+\sin \phi e^{3}, & \hat{e}^{3}=-\sin \phi e^{2}+\cos \phi e^{3} .
\end{array}
$$

The $\hat{e}^{A}$ are related to $e^{A}$ 's by a rotation by $\theta$ in the $0-1$ plane and a rotation by $\phi$ in the 2-3 plane in the tangent space. Since from (A.32) we see that on the spinors rotations in the $0-1$ plane and 2-3 plane are generated by $\frac{1}{2} \sigma_{3} \otimes I \otimes I \otimes I \otimes I$ and $\frac{1}{2} I \otimes \sigma_{3} \otimes I \otimes I \otimes I$ respectively, the rotation (A.40) is represented by the matrix

$$
\left(\begin{array}{cc}
e^{i \theta / 2} & \\
& e^{-i \theta / 2}
\end{array}\right) \otimes\left(\begin{array}{cc}
e^{i \phi / 2} & \\
& e^{-i \phi / 2}
\end{array}\right) \otimes I \otimes I \otimes I .
$$

Applying this on (A.37) and using (A.36) we get the Killing spinors in the new frame:

$$
\hat{\zeta}_{+}^{++}:\left(\begin{array}{c}
A_{1} \\
A_{2} \\
A_{3} \\
A_{4}
\end{array}\right)=N\left(\begin{array}{c}
z w \\
-z \\
-w \\
1
\end{array}\right), \quad \hat{\zeta}_{+}^{+-}:\left(\begin{array}{c}
A_{1} \\
A_{2} \\
A_{3} \\
A_{4}
\end{array}\right)=N\left(\begin{array}{c}
-w \\
1 \\
-\bar{z} w \\
\bar{z}
\end{array}\right),
$$




$$
\begin{aligned}
& \hat{\zeta}_{-}^{++}:\left(\begin{array}{c}
A_{1} \\
A_{2} \\
A_{3} \\
A_{4}
\end{array}\right)=N\left(\begin{array}{c}
-z \\
z \bar{w} \\
1 \\
-\bar{w}
\end{array}\right), \quad \hat{\zeta}_{-}^{+-}:\left(\begin{array}{c}
A_{1} \\
A_{2} \\
A_{3} \\
A_{4}
\end{array}\right)=N\left(\begin{array}{c}
1 \\
-\bar{w} \\
\bar{z} \\
-\bar{z} \bar{w}
\end{array}\right), \\
& N \equiv \frac{2 v^{1 / 4}}{\sqrt{(1+\bar{z} z)(1-\bar{w} w)}} .
\end{aligned}
$$

Similar expressions are obtained for $\zeta_{\beta}^{-\alpha}$ by replacing the $A_{i}$ 's by $B_{i}$ 's. Eq. (A.42) shows that all the Killing spinors are regular at $z=0$ and / or $w=0$.

If $\theta_{\alpha \beta}^{\gamma}$ denotes a grassman parameter labelling the supersymmetry transformations, then the supersymmetry transformation by the spinor parameter $\epsilon=\theta_{\alpha \beta}^{\gamma} \zeta_{\gamma}^{\alpha \beta}$ can be identified as the action of $i \theta_{\alpha \beta}^{\gamma} \widehat{G}_{\gamma}^{\alpha \beta}$ on various fields. Using the known supersymmetry transformation rules for various fields given in (A.19) and the definition (A.18) of $\bar{\epsilon}$ one finds

$$
\delta_{\epsilon_{2}} \delta_{\epsilon_{1}}-\delta_{\epsilon_{1}} \delta_{\epsilon_{2}}=\epsilon_{2}^{T} C \Omega \Gamma^{M} \epsilon_{1} \partial_{M},
$$

up to possible gauge transformations of the type given in (A.6). Using this we can verify that commutator of these supersymmetry generators with themselves and the other symmetries follow the $s u(1,1 \mid 2)$ algebra given in (2.13).

\section{References}

[1] A. Sen, "Quantum Entropy Function from $\operatorname{AdS}(2) / \mathrm{CFT}(1)$ Correspondence," arXiv:0809.3304 [hep-th].

[2] N. Banerjee, D. P. Jatkar and A. Sen, "Asymptotic Expansion of the N=4 Dyon Degeneracy," arXiv:0810.3472 [hep-th].

[3] A. Sen, "Arithmetic of Quantum Entropy Function," arXiv:0903.1477 [hep-th].

[4] D. Astefanesei, K. Goldstein, R. P. Jena, A. Sen and S. P. Trivedi, "Rotating attractors," JHEP 0610, 058 (2006) arXiv:hep-th/0606244.

[5] A. Sen, "Entropy Function and $A d S_{2} / C F T_{1}$ Correspondence," arXiv:0805.0095v4 [hepth].

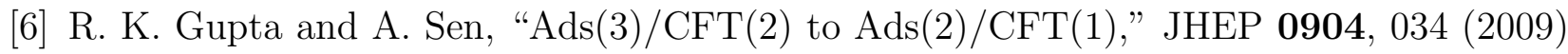
arXiv:0806.0053 [hep-th]]. 
[7] R. M. Wald, "Black hole entropy in the Noether charge," Phys. Rev. D 48, 3427 (1993) arXiv:gr-qc/9307038].

[8] T. Jacobson, G. Kang and R. C. Myers, "On Black Hole Entropy," Phys. Rev. D 49, 6587 (1994) arXiv:gr-qc/9312023].

[9] V. Iyer and R. M. Wald, "Some properties of Noether charge and a proposal for dynamical black hole entropy," Phys. Rev. D 50, 846 (1994) arXiv:gr-qc/9403028].

[10] T. Jacobson, G. Kang and R. C. Myers, "Black hole entropy in higher curvature gravity," arXiv:gr-qc/9502009.

[11] A. Sen, "Black hole entropy function and the attractor mechanism in higher derivative gravity," JHEP 0509, 038 (2005) arXiv:hep-th/0506177.

[12] A. Sen, "Black Hole Entropy Function, Attractors and Precision Counting of Microstates," arXiv:0708.1270 [hep-th].

[13] J. J. Duistermaat and G. J. Heckman, "On The Variation In The Cohomology Of The Symplectic Form Of The Reduced Phase Space," Invent. Math. 69, 259 (1982).

[14] E. Witten, "Topological Quantum Field Theory," Commun. Math. Phys. 117, 353 (1988).

[15] E. Witten, "The N Matrix Model And Gauged WZW Models," Nucl. Phys. B 371, 191 (1992).

[16] E. Witten, "Mirror manifolds and topological field theory," arXiv:hep-th/9112056.

[17] E. Witten, "Two-dimensional gauge theories revisited," J. Geom. Phys. 9 (1992) 303 arXiv:hep-th/9204083.

[18] A. S. Schwarz and O. Zaboronsky, "Supersymmetry and localization," Commun. Math. Phys. 183, 463 (1997) arXiv:hep-th/9511112.

[19] O. Zaboronsky, "Dimensional reduction in supersymmetric field theories," J. Phys. A35, $5511(2002)$.

[20] N. A. Nekrasov, "Seiberg-Witten Prepotential From Instanton Counting," Adv. Theor. Math. Phys. 7, 831 (2004) [arXiv:hep-th/0206161]. 
[21] V. Pestun, "Localization of gauge theory on a four-sphere and supersymmetric Wilson loops," arXiv:0712.2824 [hep-th].

[22] M.F. Atiyah, Elliptic operators and compact groups. Springer-Verlag, Berlin, 1974.

[23] P. Shanahan, The atiyah-singer index theorem : An introduction, Springer-Verlag.

[24] C. Beasley, D. Gaiotto, M. Guica, L. Huang, A. Strominger and X. Yin, "Why Z(BH) = -Z(top)_**2," arXiv:hep-th/0608021.

[25] J. Gomis, T. Okuda and D. Trancanelli, "Quantum 't Hooft operators and S-duality in N=4 super Yang-Mills," arXiv:0904.4486 [hep-th].

[26] S. Murthy and B. Pioline, "A Farey tale for N=4 dyons," arXiv:0904.4253 [hep-th].

[27] S. Banerjee and A. Sen, "Duality Orbits, Dyon Spectrum and Gauge Theory Limit of Heterotic String Theory on $T^{6 "}$, arXiv:0712.0043 [hep-th].

[28] S. Banerjee and A. Sen, "S-duality Action on Discrete T-duality Invariants," arXiv:0801.0149 [hep-th].

[29] A. Dabholkar, D. Gaiotto and S. Nampuri, "Comments on the spectrum of CHL dyons," arXiv:hep-th/0702150.

[30] J. H. Schwarz and A. Sen, "Type IIA Dual Of The Six-Dimensional CHL Compactification," Phys. Lett. B 357, 323 (1995) arXiv:hep-th/9507027.

[31] R. Dijkgraaf, E. P. Verlinde and H. L. Verlinde, "Counting dyons in N=4 string theory," Nucl. Phys. B 484, 543 (1997) arXiv:hep-th/9607026.

[32] G. L. Cardoso, B. de Wit, J. Kappeli and T. Mohaupt, "Asymptotic degeneracy of dyonic $\mathrm{N}=4$ string states and black hole entropy," JHEP 0412, 075 (2004) arXiv:hep-th/0412287.

[33] D. Shih, A. Strominger and X. Yin, "Recounting dyons in N = 4 string theory," JHEP 0610, 087 (2006) arXiv:hep-th/0505094.

[34] D. Gaiotto, "Re-recounting dyons in N = 4 string theory," arXiv:hep-th/0506249. 
[35] J. R. David and A. Sen, "CHL dyons and statistical entropy function from D1-D5 system," JHEP 0611, 072 (2006) arXiv:hep-th/0605210].

[36] S. Banerjee, A. Sen and Y. K. Srivastava, "Generalities of Quarter BPS Dyon Partition Function and Dyons of Torsion Two," arXiv:0802.0544 [hep-th].

[37] S. Banerjee, A. Sen and Y. K. Srivastava, "Partition Functions of Torsion $>1$ Dyons in Heterotic String Theory on $T^{6}$," arXiv:0802.1556 [hep-th].

[38] A. Dabholkar, J. Gomes and S. Murthy, "Counting all dyons in N =4 string theory," arXiv:0803.2692 [hep-th].

[39] N. Berkovits, M. Bershadsky, T. Hauer, S. Zhukov and B. Zwiebach, "Superstring theory on $\operatorname{AdS}(2) \times \mathrm{S}(2)$ as a coset supermanifold," Nucl. Phys. B 567, 61 (2000) arXiv:hep-th/9907200.

[40] A. Kapustin, "Wilson-'t Hooft operators in four-dimensional gauge theories and Sduality," Phys. Rev. D 74, 025005 (2006) arXiv:hep-th/0501015].

[41] L. J. Romans, "Selfduality For Interacting Fields: Covariant Field Equations For SixDimensional Chiral Supergravities," Nucl. Phys. B 276, 71 (1986).

[42] F. Riccioni, "Tensor multiplets in six-dimensional $(2,0)$ supergravity," Phys. Lett. B 422, 126 (1998) arXiv:hep-th/9712176|.

[43] S. Deger, A. Kaya, E. Sezgin and P. Sundell, "Spectrum of D =6, N = 4b supergravity on AdS(3) x S(3)," Nucl. Phys. B 536, 110 (1998) [arXiv:hep-th/9804166]. 